https://doi.org/10.15407/ujpe64.3.250

M.D. TOMCHENKO

Bogolyubov Institute for Theoretical Physics, Nat. Acad. of Sci. of Ukraine

(14b, Metrolohichna Str., Kyiv 03143, Ukraine; e-mail: mtomchenko@bitp.kiev.ua)

\title{
LOW-LYING ENERGY LEVELS \\ OF A ONE-DIMENSIONAL WEAKLY INTERACTING BOSE GAS UNDER ZERO BOUNDARY CONDITIONS
}

\begin{abstract}
We diagonalize the second-quantized Hamiltonian of a one-dimensional Bose gas with a nonpoint repulsive interatomic potential and zero boundary conditions. At a weak coupling, the solutions for the ground-state energy $E_{0}$ and the dispersion law $E(k)$ coincide with the Bogoliubov solutions for a periodic system. In this case, the single-particle density matrix $F_{1}\left(x, x^{\prime}\right)$ at $T=0$ is close to the solution for a periodic system and, at $T>0$, is significantly different from it. We also obtain that the wave function $\langle\hat{\psi}(x, t)\rangle$ of the effective condensate is close to a constant $\sqrt{N_{0} / L}$ inside the system and vanishes on the boundaries (here, $N_{0}$ is the number of atoms in the effective condensate, and $L$ is the size of the system). We find the criterion of applicability of the method, according to which the method works for a finite system at very low temperature and with a weak coupling (a weak interaction or a large concentration).

Keywords: interacting bosons, Bogoliubov method, zero boundary conditions.
\end{abstract}

\section{Introduction}

In the description of uniform many-particle Bose systems, the real boundary conditions (BCs) are usually replaced by periodic ones [1-5], since this simplifies the finding of a solution. However, BCs in the Nature are usually close to zero ones. Therefore, it is of significance to solve the problem for zero BCs. Such problem is of interest and not quite trivial from the mathematical point of view. From the physical viewpoint, it is very important whether the boundaries affect the bulk properties of the system such as the ground-state energy $E_{0}$, dispersion law $E(k)$, the condensate, and thermodynamic parameters. It is usually assumed that no influence is present. However, such influence is possible and, apparently, does not contradict any physical laws [6]. The solutions for a Bose system under zero BCs were found for a point interaction [7-9]. According to those results, $E_{0}$ and $E(k)$ of a one-dimensional (1D) system under zero

(C) M.D. TOMCHENKO, 2019

250
BCs differ from $E_{0}$ and $E(k)$ of a system under periodic BCs only by a negligible surface correction. For a nonpoint interaction, the problem was solved in the Haldane's harmonic-fluid approach [10,11]. It was found that the sound velocity in a system under zero BCs is the same as in a system under periodic BCs [11]. The attempt was made [6] to determine $E_{0}$ and $E(k)$ beyond the harmonic-fluid approximation. However, this calculation contains the following weak place. The ground-state wave function of the system was sought in the form $\Psi_{0}=$ $e^{S} \prod_{j=1}^{N} \sin \left(\pi x_{j} / L\right)$ (for $\left.1 \mathrm{D}\right)$, and then the equation for $S$ was solved. In this case, zero BCs hold automatically. Therefore, the differential equation for $S$ is not accompanied by $\mathrm{BCs}$ and has a continuous set of solutions that consists of the infinite number of discrete collections. One of these discrete collections is the required solution of the problem. But the probability to find just it is not too high: $1 / \infty=0$. In other words, the BCs were lost on a definite step of the method [6].

ISSN 2071-0194. Ukr. J. Phys. 2019. Vol. 64, No. 3 
In the present work, a solution of the problem with zero BCs will be found for arbitrary nonpoint repulsive potential, which can be expanded in a Fourier series, on the basis of the Bogoliubov method [1]. To simplify the calculations, the $1 \mathrm{D}$ case will be considered. Though the Bogoliubov method was developed $[12-20]$, we will be guided by the original approach [1], since it combines the simplicity and ability to describe the main properties of a system.

Note that a modified Bogoliubov method was used for describing a weakly interacting Bose gas in a harmonic trap [21, 22]. Moreover, the possibility to describe a Bose liquid as the Coulomb system of electrons and nuclei is discussed in [23].

The solution obtained below is valid for a large number of particles: $N \gtrsim 1000$. The system with $N \lesssim 100$ can be described in the exactly solvable approach $[5,7-9,24]$ (for a point-like potential) or by the multiconfigurational time-dependent Hartree method for bosons [25] (for a potential of the general form).

In Section 5, we obtain a criterion of applicability of the method. According to it, the method can be used at low temperatures $T$, a small coupling constant $\gamma$, and large finite $N$. In Section 6 , we show that the single-particle density matrix $F_{1}\left(x_{1}, x_{1}+x\right)$ as a function of $x$ decreases, as $x$ increases: by a power law at $T=0$ and exponentially at $T>0$. At such properties, the term "quasicondensate" is usually used instead of the condensate (at the exponential decrease, the term quasicondensate can be applied at low temperatures, for which $F_{1}\left(x_{1}, x_{1}+x\right)$ decreases weakly). In the region of parameters, for which the method is valid, the quasicondensate is close to the true condensate. The key aspect consists in that the system should be finite. In an infinite 1D system, the condensate (quasicondensate) is impossible for $T>0[13,14,26,27]$. The difference between finite and infinite 1D systems is easily seen from formulae in $[1,27]$ : for a finite system, one needs to integrate over $|k| \in[2 \pi / L, \infty]$ instead of $|k| \in[0, \infty]$, which leads to a possibility for the condensate to exist at $T>0$. The more strict analysis of the condensation of atoms and quasiparticles in a finite system can be found, respectively, in [28] and [29].

\section{Starting Reasonings}

Consider $N$ spinless Bose particles that are located on the interval $[0, L]$ and interact via a repulsive potential of the general form $U\left(\left|x_{j}-x_{l}\right|\right)$. Our study is essentially based on the classical work by Bogoliubov [1], and we will try to conserve its notations for the convenience of a reader. The Hamiltonian reads

$\hat{H}=-\frac{\hbar^{2}}{2 m} \sum_{j=1}^{N} \frac{\partial^{2}}{\partial x_{j}^{2}}+\sum_{j<l} U\left(\left|x_{j}-x_{l}\right|\right)$,

and zero BCs are as follows:

$\hat{\psi}(0, t)=0, \quad \hat{\psi}(L, t)=0$.

Any operator $\hat{\psi}(x, t)$ satisfying Eqs. (2) can be expanded in the complete orthonormalized collection of sines:

$\hat{\psi}(x, t)=\sum_{j=1,2, \ldots, \infty} \hat{d}_{j}(t) \sqrt{2 / L} \sin \left(k_{j} x\right)$,

where $k_{j}=\pi j / L$. In the Bogoliubov work [1], the expansion $\hat{\psi}(x, t)=\frac{1}{\sqrt{L}} \sum_{j=0, \pm 1, \ldots, \pm \infty} \hat{d}_{j}(t) e^{i 2 k_{j} x}$ was used, and it was assumed that almost all atoms are in the state $j=0$ (therefore, the replacement $\hat{d}_{0} \rightarrow d_{0}$ is executed). This allows one to construct the description of weakly excited states of a uniform system with weak coupling.

For the nonuniform system, we should apply a more general approach. There are available two different definitions of a condensate for nonuniform systems. The first is grounded on the representation $[18,30,31]$

$\hat{\psi}(x, t)=\langle\hat{\psi}(x, t)\rangle+\hat{\vartheta}(x, t)$.

The system contains a condensate described by the wave function $\langle\hat{\psi}(x, t)\rangle$, if $N_{0} \equiv \int_{0}^{L} d x|\langle\hat{\psi}(x, t)\rangle|^{2} \sim$ $\sim N$. The second definition $[18,32]$ is based on the diagonal expansion of the single-particle density matrix $F_{1}\left(x, x^{\prime}\right)$ in the complete orthonormalized basis $\left\{\phi_{j}(x)\right\}$ :

$F_{1}\left(x, x^{\prime}\right)=\sum_{j=0}^{\infty} \lambda_{j} \phi_{j}^{*}\left(x^{\prime}\right) \phi_{j}(x)$.

If $\lambda_{0} \sim N$, then a condensate is present in the state $\phi_{0}(x)$. From the physical viewpoint, the primary criterion is (5), since $\lambda_{j} / N$ is equal to the probability for a particle to be in the state $\phi_{j}(x)$. Whereas criterion (4) is rather a way to use a single-particle function in the description of a many-particle system. These two criteria are equivalent for a uniform periodic system (Bogoliubov solution [1]). However, they can be 
nonequivalent for a nonuniform system [33]. Therefore, we will consider the condensate defined on the basis of criterion (5) to be genuine. In this case, we will refer the order parameter $\langle\hat{\psi}(x, t)\rangle$ to the effective condensate.

Here and below, \langle\rangle stands for the statistical average [34]:

$$
\langle\hat{A}\rangle_{T>0}=\frac{1}{Z} \int d x_{1} \ldots d x_{N} \sum_{p} e^{-E_{p} / k_{\mathrm{B}} T} \Psi_{p}^{*} \hat{A} \Psi_{p}
$$

where $Z=\sum_{l} e^{-E_{l} / k_{\mathrm{B}} T}$, and $\left\{\Psi_{p}\left(x_{1}, \ldots, x_{N}\right)\right\}$ is the complete orthonormalized set of wave functions of a system with a fixed number of particles $N$. For the pure state, which is possible in the many-particle system only at $T=0$, it is the quantum-mechanical average:

$$
\langle\hat{A}\rangle_{T=0}=\int d x_{1} \ldots d x_{N} \Psi_{0}^{*} \hat{A} \Psi_{0} .
$$

The operator $\hat{\psi}(x, t)$ can be expanded in any singleparticle basis satisfying zero BCs. In this case, the operator $\hat{\psi}(x, t)$, values of $E_{p}$, functions $\Psi_{p}$, and function $\langle\hat{\psi}(x, t)\rangle$ are independent of the basis.

Relations (3) and (4) yield

$$
\begin{aligned}
& \hat{d}_{j}(t)=\left\langle\hat{d}_{j}(t)\right\rangle+\hat{a}_{j}(t) \equiv d_{j}(t)+\hat{a}_{j}(t), \\
& \langle\hat{\psi}(x, t)\rangle=\sum_{j=1,2, \ldots, \infty} d_{j}(t) \sqrt{2 / L} \sin \left(k_{j} x\right), \\
& \hat{\vartheta}(x, t)=\sum_{l=1,2, \ldots, \infty} \hat{a}_{l}(t) \sqrt{2 / L} \sin \left(k_{l} x\right), \\
& \hat{\psi}^{+}(x, t)=\sum_{j=1,2, \ldots, \infty} \hat{d}_{j}^{+}(t) \sqrt{2 / L} \sin \left(k_{j} x\right)= \\
& =\left\langle\hat{\psi}^{+}(x, t)\right\rangle+\hat{\vartheta}^{+}(x, t), \\
& \hat{\vartheta}^{+}(x, t)=\sum_{l=1,2, \ldots, \infty} \hat{a}_{l}^{+}(t) \sqrt{2 / L} \sin \left(k_{l} x\right) .
\end{aligned}
$$

The Bose operators $\hat{d}_{l}^{+}$and $\hat{d}_{j}$ satisfy the commutation relations

$$
\begin{aligned}
& \hat{d}_{j} \hat{d}_{l}^{+}-\hat{d}_{l}^{+} \hat{d}_{j}=\delta_{l, j}, \\
& \hat{d}_{j} \hat{d}_{l}-\hat{d}_{l} \hat{d}_{j}=\hat{d}_{j}^{+} \hat{d}_{l}^{+}-\hat{d}_{l}^{+} \hat{d}_{j}^{+}=0 .
\end{aligned}
$$

The operators $\hat{a}_{l}^{+}$and $\hat{a}_{j}$ satisfy the same relations.

The subsequent analysis is based on relations (4) and $\hat{\vartheta} \ll\langle\hat{\psi}(x, t)\rangle$. They are basic formulae allowing one to construct the description of a nonuniform weakly interacting Bose gas.

\section{Method 1: Solving the Operator Equation}

The Heisenberg equation for the operator $\hat{\psi}=$ $=\langle\hat{\psi}(x, t)\rangle+\hat{\vartheta}$ reads

$i \hbar \frac{\partial \hat{\psi}(x, t)}{\partial t}=-\frac{\hbar^{2}}{2 m} \frac{\partial^{2} \hat{\psi}(x, t)}{\partial x^{2}}+$

$+\int_{0}^{L} d x^{\prime} U\left(\left|x-x^{\prime}\right|\right) \hat{\psi}^{+}\left(x^{\prime}, t\right) \hat{\psi}\left(x^{\prime}, t\right) \hat{\psi}(x, t)$.

At $\hat{\vartheta} \ll\langle\hat{\psi}(x, t)\rangle$, it can be separated into two equations (in this section, we denote $\langle\hat{\psi}(x, t)\rangle \equiv \psi_{0}(x, t)$ ):

$i \hbar \frac{\partial \psi_{0}(x, t)}{\partial t}=-\frac{\hbar^{2}}{2 m} \frac{\partial^{2} \psi_{0}(x, t)}{\partial x^{2}}+$

$+\psi_{0}(x, t) \int_{0}^{L} d x^{\prime} U\left(\left|x-x^{\prime}\right|\right)\left|\psi_{0}\left(x^{\prime}, t\right)\right|^{2}$,

$i \hbar \frac{\partial \hat{\vartheta}(x, t)}{\partial t}=-\frac{\hbar^{2}}{2 m} \frac{\partial^{2} \hat{\vartheta}(x, t)}{\partial x^{2}}+$

$+\hat{\vartheta}(x, t) \int_{0}^{L} d x^{\prime} U\left(\left|x-x^{\prime}\right|\right)\left|\psi_{0}\left(x^{\prime}, t\right)\right|^{2}+$

$+\psi_{0}(x, t) \int_{0}^{L} d x^{\prime} U\left(\left|x-x^{\prime}\right|\right)\left[\psi_{0}\left(x^{\prime}, t\right) \hat{\vartheta}^{+}\left(x^{\prime}, t\right)+\right.$

$\left.+\psi_{0}^{*}\left(x^{\prime}, t\right) \hat{\vartheta}\left(x^{\prime}, t\right)\right]$.

Equation (15) was first obtained by Gross [35] and is usually called the Gross-Pitaevskii equation [36, 37]. It is clear that the lowest energy solution of Eq. (15) is given by the function $\psi_{0}(x, t)=\psi_{0}(t)$. To satisfy zero BCs, $\psi_{0}(x, t)$ must decrease to zero near the boundaries. Neglecting this nonuniformity, we can write $\psi_{0}(x, t)=a_{0}(t) / \sqrt{L}$ (more accurate solution for $\langle\hat{\psi}(x, t)\rangle$ will be obtained in the next section). We set $a_{0}^{*} a_{0}=N_{0}$ and $n_{0}=N_{0} / L$. Then Eq. (15) takes the form

$i \hbar \frac{\partial a_{0}(t)}{\partial t}=a_{0}(t) n_{0} \int_{0}^{L} d x^{\prime} U\left(\left|x-x^{\prime}\right|\right)$.

We expand the potential in the Fourier series:

$U\left(\left|x_{1}-x_{2}\right|\right)=\frac{1}{2 L} \sum_{j=0, \pm 1, \pm 2, \ldots} \nu\left(k_{j}\right) e^{i k_{j}\left(x_{1}-x_{2}\right)}$, 


$$
\nu\left(k_{j}\right)=\int_{-L}^{L} U(|x|) e^{-i k_{j} x} d x, \quad k_{j}=\pi j / L .
$$

This series restores the potential exactly in the whole region $x_{1}, x_{2} \in[0, L]$ (expansions of the potential for different BCs have been considered in [38]). Since $\nu\left(k_{j}\right)=2 \int_{0}^{L} U(x) \cos \left(k_{j} x\right) d x=\nu\left(-k_{j}\right)$, we have

$U\left(\left|x-x^{\prime}\right|\right)=\frac{\nu(0)}{2 L}+\sum_{j=1,2, \ldots} \frac{\nu\left(k_{j}\right)}{L} \times$
$\times\left[\cos \left(k_{j} x\right) \cos \left(k_{j} x^{\prime}\right)+\sin \left(k_{j} x\right) \sin \left(k_{j} x^{\prime}\right)\right]$.

Substituting expansion (20) in (17) and making some transformations, we get the equation

$i \hbar \frac{\partial a_{0}(t)}{\partial t}=a_{0}(t) n_{0} \nu(0)\left(1+S_{1}(x)\right)$.

The function $S_{1}(x)$ and the functions $S_{j}(x)$ arising below are given and calculated in Appendix B. If the interaction radius $a$ is small as compared with the system size $L$, then all functions $S_{j}(x)$ are negligibly small. We find the solution of Eq. (21) as

$a_{0}(t)=e^{\epsilon_{0} t / i \hbar} b_{0}, \quad \epsilon_{0}=n_{0} \nu(0)$.

In view of zero BCs, the solution for $\psi_{0}$ can be written in the form (9):

$\psi_{0}(x, t)=\frac{a_{0}(t)}{\sqrt{L}} \sum_{j=0,1, \ldots, \infty} \frac{4 \sin \left(k_{2 j+1} x\right)}{\pi(2 j+1)}=$
$=\left[\begin{array}{ll}\frac{a_{0}(t)}{\sqrt{L}}, & \text { if } x \in] 0, L[, \\ 0, & \text { if } x=0 ; L .\end{array}\right.$

Now, let us substitute $\hat{\vartheta}(10)$ and $\hat{\vartheta}^{+}(12)$ in Eq. (16). With regard for relations (20) and (23), Eq. (16) is separated into two equations for the harmonics $\hat{a}_{2 l}$ and $\hat{a}_{2 j+1}$. The equation for $\hat{a}_{2 l}$ reads

$$
\begin{aligned}
& i \hbar \sum_{l=1,2, \ldots, \infty} \sin \left(k_{2 l} x\right) \frac{\partial \hat{a}_{2 l}(t)}{\partial t}=\sum_{l=1,2, \ldots, \infty} \sin \left(k_{2 l} x\right) \times \\
& \times \hat{a}_{2 l}(t)\left(K\left(k_{2 l}\right)+n_{0} \nu(0)\left[1+S_{1}(x)\right]\right)+ \\
& +\sum_{l=1,2, \ldots, \infty}\left(n_{0} \hat{a}_{2 l}(t)+\frac{a_{0}^{2}}{L} \hat{a}_{2 l}^{+}(t)\right) \times \\
& \times\left(\frac{\nu\left(k_{2 l}\right)}{2} \sin \left(k_{2 l} x\right)+I_{1}(x, l)\right),
\end{aligned}
$$

$I_{1}(x, l)=\sum_{j=1,3,5, \ldots} \frac{\nu\left(k_{j}\right)}{\pi} \cos \left(k_{j} x\right)\left(\frac{1}{2 l-j}+\frac{1}{2 l+j}\right)$

where $K(k)=\frac{\hbar^{2} k^{2}}{2 m}$. Using the expansion

$\cos \left(k_{j} x\right)=\sum_{p=1,2,3, \ldots} c_{j}^{p} \sin \left(k_{p} x\right)$,

$c_{j}^{p}=\left[\begin{array}{ll}0 & \text { for even } p-j, \\ \frac{2}{\pi}\left(\frac{1}{p-j}+\frac{1}{p+j}\right) & \text { for odd } p-j,\end{array}\right.$

the sum $I_{1}(25)$ can be represented in the form

$I_{1}(x, l)=\nu\left(k_{2 l}\right) \sin \left(k_{2 l} x\right)\left(\frac{1}{2}+S_{2}(l)\right)+$

$+\frac{2}{\pi^{2}} \sum_{j=1,3,5, \ldots} \nu\left(k_{j}\right) \sum_{p=1,2,3, \ldots}^{p \neq l} \sin \left(k_{2 p} x\right) \times$

$\times\left(\frac{1}{2 l-j}+\frac{1}{2 l+j}\right)\left(\frac{1}{2 p-j}+\frac{1}{2 p+j}\right)$.

Then Eq. (24) takes the form

$i \hbar \sum_{l=1,2, \ldots, \infty} \sin \left(k_{2 l} x\right) \frac{\partial \hat{a}_{2 l}(t)}{\partial t}=$

$=\sum_{l=1,2, \ldots, \infty} \sin \left(k_{2 l} x\right) \frac{a_{0}^{2}}{L} \nu\left(k_{2 l}\right) \hat{a}_{2 l}^{+}(t)\left(1+S_{2}(l)\right)+$

$+\sum_{l=1,2, \ldots, \infty} \sin \left(k_{2 l} x\right) \hat{a}_{2 l}(t)\left[K\left(k_{2 l}\right)+\right.$

$\left.+n_{0} \nu(0)\left(1+S_{1}(x)\right)+n_{0} \nu\left(k_{2 l}\right)\left(1+S_{2}(l)\right)\right]+$

$+\sum_{l=1,2, \ldots, \infty} \sin \left(k_{2 l} x\right) \sum_{p=1,2, \ldots, \infty}^{p \neq l}\left(n_{0} \hat{a}_{2 p}(t)+\right.$

$\left.+\frac{a_{0}^{2}}{L} \hat{a}_{2 p}^{+}(t)\right) S_{3}(l, p)$.

Since the functions $\sin \left(k_{2 l} x\right)$ are independent, and the corrections $S_{1}, S_{2}$, and $S_{3}$ are vanishingly small (see Appendix B), Eq. (28) yields the system of equations $i \hbar \frac{\partial \hat{a}_{2 l}(t)}{\partial t}=\left[K\left(k_{2 l}\right)+n_{0} \nu(0)+n_{0} \nu\left(k_{2 l}\right)\right] \hat{a}_{2 l}(t)+$ $+\frac{a_{0}^{2} \nu\left(k_{2 l}\right)}{L} \hat{a}_{2 l}^{+}(t), \quad l=1,2, \ldots, \infty$.

In a similar way, we find the following equation for the operators $\hat{a}_{2 j+1}$ from (16):

$i \hbar \sum_{j=0,1, \ldots, \infty} \sin \left(k_{2 j+1} x\right) \frac{\partial \hat{a}_{2 j+1}(t)}{\partial t}=$ 


$$
\begin{aligned}
& =\sum_{j=0,1, \ldots, \infty} \sin \left(k_{2 j+1} x\right) \frac{a_{0}^{2}}{L} \nu\left(k_{2 j+1}\right) \times \\
& \times \hat{a}_{2 j+1}^{+}(t)\left(1+S_{4}(j)\right)+ \\
& +\sum_{j=0,1, \ldots, \infty} \sin \left(k_{2 j+1} x\right) \hat{a}_{2 j+1}(t)\left[K\left(k_{2 j+1}\right)+\right. \\
& \left.+n_{0} \nu(0)\left(1+S_{1}(x)\right)+n_{0} \nu\left(k_{2 j+1}\right)\left(1+S_{4}(j)\right)\right]+ \\
& +\sum_{j=0,1, \ldots, \infty} \sin \left(k_{2 j+1} x\right) \times \\
& \times \sum_{l=0,1, \ldots, \infty}^{l \neq j}\left(n_{0} \hat{a}_{2 l+1}(t)+\frac{a_{0}^{2}}{L} \hat{a}_{2 l+1}^{+}(t)\right) S_{5}(l, j) .
\end{aligned}
$$

Since the corrections $S_{1}, S_{4}, S_{5}$ are small, and the functions $\sin \left(k_{2 j+1} x\right)$ are independent, Eq. (30) leads to the equations

$i \hbar \frac{\partial \hat{a}_{2 j+1}(t)}{\partial t}=\frac{a_{0}^{2} \nu\left(k_{2 j+1}\right)}{L} \hat{a}_{2 j+1}^{+}(t)+$
$+\left[K\left(k_{2 j+1}\right)+n_{0} \nu(0)+n_{0} \nu\left(k_{2 j+1}\right)\right] \hat{a}_{2 j+1}(t)$,

where $j=0,1, \ldots, \infty$.

Thus, the starting equation (14) is reduced to Eqs. (21), (29), and (31). Equations (29) and (31) coincide with the equation for $\hat{a}_{p}$ from the Bogoliubov work [1] with the only difference that our equations involve $\hat{a}_{p}^{+}$instead of $\hat{a}_{-p}^{+}$in [1]. This allows us to write the solution immediately. We set $\hat{a}_{p}(t)=$ $=e^{\epsilon_{0} t / i \hbar} \hat{b}_{p}(p=1,2, \ldots, \infty)$ and $a_{0}(t)=e^{\epsilon_{0} t / i \hbar} b_{0}$. With the help of Bogoliubov transformations

$\hat{b}_{p}=\frac{\hat{\xi}_{p}+\Lambda_{p} \hat{\xi}_{p}^{+}}{\sqrt{1-\left|\Lambda_{p}\right|^{2}}}, \quad \hat{b}_{p}^{+}=\frac{\hat{\xi}_{p}^{+}+\Lambda_{p}^{*} \hat{\xi}_{p}}{\sqrt{1-\left|\Lambda_{p}\right|^{2}}}$,

$\hat{\xi}_{p}=\frac{\hat{b}_{p}-\Lambda_{p} \hat{b}_{p}^{+}}{\sqrt{1-\left|\Lambda_{p}\right|^{2}}}, \quad \hat{\xi}_{p}^{+}=\frac{\hat{b}_{p}^{+}-\Lambda_{p}^{*} \hat{b}_{p}}{\sqrt{1-\left|\Lambda_{p}\right|^{2}}}$,

$\Lambda_{p}=\frac{b_{0}^{2}}{N_{0} n_{0} \nu\left(k_{p}\right)}\left(E_{b}\left(k_{p}\right)-K\left(k_{p}\right)-n_{0} \nu\left(k_{p}\right)\right)$,

we obtain the following equations from (29) and (31):

$i \hbar \frac{\partial \hat{\xi}_{p}}{\partial t}=E_{b}\left(k_{p}\right) \hat{\xi}_{p}, \quad-i \hbar \frac{\partial \hat{\xi}_{p}^{+}}{\partial t}=E_{b}\left(k_{p}\right) \hat{\xi}_{p}^{+}$.

Here, $p=1,2, \ldots, \infty$ and

$E_{b}(k)=\sqrt{K^{2}(k)+2 n_{0} \nu(k) K(k)}$.

254
Our solution coincides with the Bogoliubov one [1] except for the following difference: in the Bogoliubov formulae (35), $p$ runs the values $\pm 1, \pm 2, \ldots, \pm \infty$; whereas our $p$ are positive $(p=1,2, \ldots, \infty)$, since the excitations are standing waves. Formulae (35) show that the excited state of the system can be considered as an ideal gas of elementary excitations with the dispersion law $E_{b}(k)(36)$.

\section{Method 2: Diagonalization of the Hamiltonian}

In the second-quantized formalism, Hamiltonian (1) takes the form [34]

$\hat{H}=\hat{H}_{\text {kin }}+\hat{H}_{\text {pot }}$,

$\hat{H}_{\text {kin }}=-\frac{\hbar^{2}}{2 m} \int_{0}^{L} d x \hat{\psi}^{+}(x, t) \frac{\partial^{2}}{\partial x^{2}} \hat{\psi}(x, t)$,

$\hat{H}_{\text {pot }}=\frac{1}{2} \int_{0}^{L} d x d x^{\prime} U\left(\left|x-x^{\prime}\right|\right) \hat{\psi}^{+}(x, t) \hat{\psi}^{+}\left(x^{\prime}, t\right) \times$

$\times \hat{\psi}(x, t) \hat{\psi}\left(x^{\prime}, t\right)$.

We set $\hat{\psi}(x, t)=\langle\hat{\psi}(x, t)\rangle+\hat{\vartheta}(x, t)$, where $\langle\hat{\psi}(x, t)\rangle=\psi_{0}(x, t)+\delta \psi_{0}(x, t)$. Here, $\psi_{0}(x, t)=$ $=\sum_{j=1,2, \ldots, \infty} d_{j}^{(0)}(t) \sqrt{2 / L} \sin \left(k_{j} x\right)$ is the bare part of the function $\langle\hat{\psi}(x, t)\rangle$ and is given by formula (23), and $\delta \psi_{0}(x, t)$ is an unknown small correction. First, we set $\delta \psi_{0}(x, t)=0$. Then $\delta \psi_{0}(x, t)$ will be determined from the analysis. In this case, while calculating $\left(\partial^{2} / \partial x^{2}\right) \hat{\psi}(x)$, we should take into account that the function $\psi_{0}(x)(23)$ is constant for $\left.x \in\right] 0, L[$ and decreases to zero by jump at the points $x=0$ and $L$. Instead, it is simpler to use the initial exact expansion (3). In this case, we note that (23) yields

$d_{2 j}^{(0)}=0, \quad j=1,2, \ldots, \infty$.

In such way with regard for the relations $\hat{d}_{j}(t)=$ $=d_{j}^{(0)}(t)+\hat{a}_{j}(t), \hat{a}_{j}(t)=e^{\epsilon_{0} t / i \hbar} \hat{b}_{j}$, and (40), we obtain

$$
\begin{aligned}
& \hat{H}_{\text {kin }}=\sum_{l=1,2, \ldots, \infty} K\left(k_{2 l}\right) \hat{b}_{2 l}^{+} \hat{b}_{2 l}+ \\
& +\sum_{j=0,1, \ldots, \infty} K\left(k_{2 j+1}\right)\left(\hat{b}_{2 j+1}^{+}+f_{2 j+1}^{*}\right) \times \\
& \times\left(\hat{b}_{2 j+1}+f_{2 j+1}\right),
\end{aligned}
$$


where

$f_{2 j+1}=d_{2 j+1}^{(0)} e^{-\epsilon_{0} t / i \hbar}=\frac{2 \sqrt{2} b_{0}}{k_{2 j+1} L}$.

The operator $\hat{H}_{\text {pot }}$ contains no derivatives. Therefore, we use the representation $\hat{\psi}=\psi_{0}+\hat{\vartheta}$ with $\psi_{0}$ (23). Similarly to the Bogoliubov work, we neglect the terms of the 3 -rd and 4 -th degrees in $\hat{\vartheta}$ and $\hat{\vartheta}^{+}$in the product $\hat{\psi}^{+}(x) \hat{\psi}^{+}\left(x^{\prime}\right) \hat{\psi}(x) \hat{\psi}\left(x^{\prime}\right)$. Then

$$
\begin{aligned}
& \hat{\psi}^{+}(x) \hat{\psi}^{+}\left(x^{\prime}\right) \hat{\psi}(x) \hat{\psi}\left(x^{\prime}\right)=\left.\frac{\left(a_{0} a_{0}^{*}\right)^{2}}{L^{2}}\right|_{1}+ \\
& +\left.\frac{a_{0}^{*} a_{0}^{2}}{L^{3 / 2}}\left(\hat{\vartheta}^{+}(x)+\hat{\vartheta}^{+}\left(x^{\prime}\right)\right)\right|_{3}+\left.\frac{\left(a_{0}^{*}\right)^{2}}{L} \hat{\vartheta}(x) \hat{\vartheta}\left(x^{\prime}\right)\right|_{4}+ \\
& +\left.\frac{a_{0}\left(a_{0}^{*}\right)^{2}}{L^{3 / 2}}\left(\hat{\vartheta}(x)+\hat{\vartheta}\left(x^{\prime}\right)\right)\right|_{2}+\left.\frac{a_{0}^{2}}{L} \hat{\vartheta}^{+}(x) \hat{\vartheta}^{+}\left(x^{\prime}\right)\right|_{5}+ \\
& +\left.\frac{a_{0} a_{0}^{*}}{L}\left(\hat{\vartheta}^{+}(x)+\hat{\vartheta}^{+}\left(x^{\prime}\right)\right)\left(\hat{\vartheta}(x)+\hat{\vartheta}\left(x^{\prime}\right)\right)\right|_{6} \equiv \\
& \equiv \sum_{l=1,2, \ldots, 6} \hat{\eta}_{l} .
\end{aligned}
$$

Expression (43) is a sum of six terms. Each of them is enumerated by the mark $\left.\right|_{l}$ and is denoted by $\hat{\eta}_{l}$. Using expansion (20) of the potential, we get

$$
\begin{aligned}
& \hat{H}_{\mathrm{pot}}=\sum_{l=1,2, \ldots, 6} \hat{I}_{l}, \\
& \hat{I}_{l}=\frac{1}{2} \int_{0}^{L} d x d x^{\prime} \hat{\eta}_{l}\left\{\frac{\nu(0)}{2 L}+\sum_{j=1,2, \ldots} \frac{\nu\left(k_{j}\right)}{L} \times\right. \\
& \left.\times\left[\cos \left(k_{j} x\right) \cos \left(k_{j} x^{\prime}\right)+\sin \left(k_{j} x\right) \sin \left(k_{j} x^{\prime}\right)\right]\right\} .
\end{aligned}
$$

The calculation of integrals (45) gives rather awkward sums, and the main problem consists in the separation of their principal parts. With regard for formulae (9)-(12), (42), $a_{0}(t)=e^{\epsilon_{0} t / i \hbar} b_{0}$, and $b_{0}^{*} b_{0}=N_{0}$, we obtain

$$
\begin{aligned}
& \hat{I}_{1}=\frac{N_{0} n_{0} \nu(0)}{2}\left(1+S_{21}\right), \\
& \hat{I}_{2}=\frac{n_{0}}{2} \sum_{j=0,1, \ldots, \infty} f_{2 j+1}^{*} \hat{b}_{2 j+1}\left(\nu(0)+\nu\left(k_{2 j+1}\right)\right), \\
& \hat{I}_{3}=\frac{n_{0}}{2} \sum_{j=0,1, \ldots, \infty} f_{2 j+1} \hat{b}_{2 j+1}^{+}\left(\nu(0)+\nu\left(k_{2 j+1}\right)\right), \\
& \hat{I}_{4}=\frac{\left(b_{0}^{*}\right)^{2}}{2 L} \sum_{l=1,2, \ldots, \infty} \nu\left(k_{2 l}\right) \hat{b}_{2 l}^{2}\left(1+S_{22}(l)\right)+
\end{aligned}
$$

$$
\begin{aligned}
& +\sum_{j=0,1, \ldots, \infty} \nu\left(k_{2 j+1}\right) \hat{b}_{2 j+1}^{2}\left(1+S_{23}(j)\right)+ \\
& +\sum_{l_{1}, l_{2}=1,2, \ldots, \infty}^{l_{1} \neq l_{2}} \hat{b}_{2 l_{1}} \hat{b}_{2 l_{2}} S_{24}\left(l_{1}, l_{2}\right)+ \\
& \left.+\sum_{j_{1}, j_{2}=0,1, \ldots, \infty}^{j_{1} \neq j_{2}} \hat{b}_{2 j_{1}+1} \hat{b}_{2 j_{2}+1} S_{25}\left(j_{1}, j_{2}\right)\right] \text {, } \\
& \hat{I}_{5}=\frac{b_{0}^{2}}{2 L}\left[\sum_{l=1,2, \ldots, \infty} \nu\left(k_{2 l}\right)\left(\hat{b}_{2 l}^{+}\right)^{2}\left(1+S_{22}(l)\right)+\right. \\
& +\sum_{j=0,1, \ldots, \infty} \nu\left(k_{2 j+1}\right)\left(\hat{b}_{2 j+1}^{+}\right)^{2}\left(1+S_{23}(j)\right)+ \\
& +\sum_{l_{1}, l_{2}=1,2, \ldots, \infty}^{l_{1} \neq l_{2}} \hat{b}_{2 l_{1}}^{+} \hat{b}_{2 l_{2}}^{+} S_{24}\left(l_{1}, l_{2}\right)+ \\
& \left.+\sum_{j_{1}, j_{2}=0,1, \ldots, \infty}^{j_{1} \neq j_{2}} \hat{b}_{2 j_{1}+1}^{+} \hat{b}_{2 j_{2}+1}^{+} S_{25}\left(j_{1}, j_{2}\right)\right] \text {, } \\
& \hat{I}_{6}=\frac{(N-\hat{\tilde{N}}) n_{0} \nu(0)}{2}+ \\
& +\sum_{l=1,2, \ldots, \infty} n_{0} \nu\left(k_{2 l}\right) \hat{b}_{2 l}^{+} \hat{b}_{2 l}\left(1+S_{22}(l)+\tilde{S}_{26}(l)\right)+ \\
& +\sum_{j=0,1, \ldots, \infty} n_{0} \nu\left(k_{2 j+1}\right) \hat{b}_{2 j+1}^{+} \hat{b}_{2 j+1} \times \\
& \times\left(1+S_{23}(j)+\tilde{S}_{27}(j)\right)+ \\
& +\sum_{l_{1}, l_{2}=1,2, \ldots, \infty}^{l_{1} \neq l_{2}} n_{0} \hat{b}_{2 l_{1}}^{+} \hat{b}_{2 l_{2}}\left[S_{24}\left(l_{1}, l_{2}\right)+S_{28}\left(l_{1}, l_{2}\right)\right]+ \\
& +\sum_{j_{1}, j_{2}=0,1, \ldots, \infty}^{j_{1} \neq j_{2}} n_{0} \hat{b}_{2 j_{1}+1}^{+} \hat{b}_{2 j_{2}+1} \times \\
& \times\left[S_{25}\left(j_{1}, j_{2}\right)+S_{29}\left(j_{1}, j_{2}\right)\right],
\end{aligned}
$$

where we denote

$N-\hat{\tilde{N}} \equiv \sum_{l=1,2, \ldots, \infty} \hat{b}_{2 l}^{+} \hat{b}_{2 l}+\sum_{j=0,1, \ldots, \infty} \hat{b}_{2 j+1}^{+} \hat{b}_{2 j+1}$.

Using the relations $\hat{b}_{l}=e^{-\epsilon_{0} t / i \hbar} \hat{a}_{l}=e^{-\epsilon_{0} t / i \hbar}\left(\hat{d}_{l}-d_{l}^{(0)}\right)$, (40) and the normalization condition $\sum_{l=1,2, \ldots, \infty} \hat{d}_{l}^{+} \hat{d}_{l}=N$, we represent (52) in the form

$N-\hat{\tilde{N}}=N-\sum_{j=0,1, \ldots, \infty}\left|f_{2 j+1}\right|^{2}-$
$-\sum_{j=0,1, \ldots, \infty}\left(\hat{b}_{2 j+1}^{+} f_{2 j+1}+\hat{b}_{2 j+1} f_{2 j+1}^{*}\right)$. 
It is shown in Appendix B that $\tilde{S}_{26}(l)=\frac{\nu(0)}{2 \nu\left(k_{2 l}\right)}-$ $-S_{26}(l), \tilde{S}_{27}(j)=\frac{\nu(0)}{2 \nu\left(k_{2 j+1}\right)}-S_{27}(j)$, and the corrections $S_{2 j}$ with $j=1,2, \ldots, 9$ are negligibly small at $a \ll L$. Taking this into account, we find from Eqs. (37)-(53):

$$
\begin{aligned}
& \hat{H}=\hat{H}_{\text {lin }}+\frac{N_{0} n_{0} \nu(0)}{2}+N n_{0} \nu(0)+ \\
& +\sum_{j=0,1, \ldots, \infty}\left(K\left(k_{2 j+1}\right)-n_{0} \nu_{0}\right)\left|f_{2 j+1}\right|^{2}+ \\
& +\sum_{l=1,2, \ldots, \infty}\left[\left(K\left(k_{2 l}\right)+n_{0} \nu\left(k_{2 l}\right)\right) \hat{b}_{2 l}^{+} \hat{b}_{2 l}+\right. \\
& \left.+\frac{\left(b_{0}^{*}\right)^{2}}{2 L} \nu\left(k_{2 l}\right) \hat{b}_{2 l}^{2}+\frac{b_{0}^{2}}{2 L} \nu\left(k_{2 l}\right)\left(\hat{b}_{2 l}^{+}\right)^{2}\right]+ \\
& +\sum_{j=0,1, \ldots}\left[\left(K\left(k_{2 j+1}\right)+n_{0} \nu\left(k_{2 j+1}\right)\right) \hat{b}_{2 j+1}^{+} \hat{b}_{2 j+1}+\right. \\
& \left.+\frac{\left(b_{0}^{*}\right)^{2}}{2 L} \nu\left(k_{2 j+1}\right) \hat{b}_{2 j+1}^{2}+\frac{b_{0}^{2}}{2 L} \nu\left(k_{2 j+1}\right)\left(\hat{b}_{2 j+1}^{+}\right)^{2}\right], \\
& \hat{H}_{\text {lin }}=\sum_{j=0,1, \ldots, \infty}\left(f_{2 j+1}^{*} \hat{b}_{2 j+1}+f_{2 j+1} \hat{b}_{2 j+1}^{+}\right) \times \\
& \times\left[K\left(k_{2 j+1}\right)+n_{0} \nu\left(k_{2 j+1}\right) / 2-n_{0} \nu_{0} / 2\right] .
\end{aligned}
$$

The correction term $\hat{H}_{\text {lin }}$ is linear in $\hat{b}_{2 j+1}$ and $\hat{b}_{2 j+1}^{+}$ and can be removed with the help of the transformation

$$
\begin{aligned}
& \hat{b}_{2 j+1}=\hat{\tilde{b}}_{2 j+1}+\beta_{2 j+1}, \quad \hat{b}_{2 j+1}^{+}=\hat{\tilde{b}}_{2 j+1}^{+}+\beta_{2 j+1}^{*}, \\
& \beta_{2 j+1}=-f_{2 j+1} \frac{2 K\left(k_{2 j+1}\right)+n_{0} \nu\left(k_{2 j+1}\right)-n_{0} \nu_{0}}{2 K\left(k_{2 j+1}\right)+4 n_{0} \nu\left(k_{2 j+1}\right)},
\end{aligned}
$$

where $j=0,1, \ldots, \infty$. The operators $\hat{\tilde{b}}_{2 j+1}^{+}$and $\hat{\tilde{b}}_{2 p+1}$ satisfy the same commutation relations as the operators $\hat{b}_{2 j+1}^{+}$and $\hat{b}_{2 p+1}$. Thus, we have found the correction $\delta \psi_{0}(x, t)$ :

$$
\begin{aligned}
& \delta \psi_{0}(x, t) \equiv\langle\hat{\psi}(x, t)\rangle-\psi_{0}(x, t)= \\
& =e^{\epsilon_{0} t / i \hbar} \sum_{j=0,1, \ldots, \infty} \beta_{2 j+1} \sqrt{2 / L} \sin \left(k_{2 j+1} x\right) .
\end{aligned}
$$

In the previous section, the correction $\beta_{2 j+1}$ did not arise, because a more approximate solution was found. Let us substitute the operators $\hat{b}_{2 j+1}$ and $\hat{b}_{2 j+1}^{+}$ (56) in Eqs. (54), (55). After some transformations, we obtain the following total Hamiltonian:

$\hat{H}=\frac{N_{0} n_{0} \nu(0)}{2}+\left(N-N_{0}\right) n_{0} \nu(0)+\delta E_{0}+$

$$
\begin{aligned}
& +\sum_{l=1,2, \ldots, \infty}\left[\left(K\left(k_{2 l}\right)+n_{0} \nu\left(k_{2 l}\right)\right) \hat{b}_{2 l}^{+} \hat{b}_{2 l}+\right. \\
& \left.+\frac{\left(b_{0}^{*}\right)^{2}}{2 L} \nu\left(k_{2 l}\right) \hat{b}_{2 l}^{2}+\frac{b_{0}^{2}}{2 L} \nu\left(k_{2 l}\right)\left(\hat{b}_{2 l}^{+}\right)^{2}\right]+ \\
& +\sum_{j=0,1, \ldots, \infty}\left[\left(K\left(k_{2 j+1}\right)+n_{0} \nu\left(k_{2 j+1}\right)\right) \times\right. \\
& \times \hat{\tilde{b}}_{2 j+1}^{+} \hat{\tilde{b}}_{2 j+1}+\frac{\left(b_{0}^{*}\right)^{2}}{2 L} \nu\left(k_{2 j+1}\right) \hat{\tilde{b}}_{2 j+1}^{2}+ \\
& \left.+\frac{b_{0}^{2}}{2 L} \nu\left(k_{2 j+1}\right)\left(\hat{\tilde{b}}_{2 j+1}^{+}\right)^{2}\right], \\
& \delta E_{0}=N_{0} n_{0} \nu(0)+ \\
& +\sum_{j=0,1, \ldots, \infty}\left|f_{2 j+1}\right|^{2}\left(K\left(k_{2 j+1}\right)-n_{0} \nu_{0}\right)- \\
& -\sum_{j=0,1, \ldots, \infty}\left|\beta_{2 j+1}\right|^{2}\left(K\left(k_{2 j+1}\right)+2 n_{0} \nu\left(k_{2 j+1}\right)\right) .
\end{aligned}
$$

With the help of transforms (32), where we use the operators $\hat{b}_{2 l}^{+}, \hat{b}_{2 l}$ for even $p$ and the operators $\hat{\tilde{b}}_{2 j+1}^{+}, \hat{\tilde{b}}_{2 j+1}$ for odd $p$, Hamiltonian (59) is reduced to the diagonal form:

$$
\begin{aligned}
& \hat{H}=E_{0}+\sum_{l=1,2, \ldots, \infty} E_{b}\left(k_{l}\right) \hat{\xi}_{l}^{+} \hat{\xi}_{l}, \\
& E_{0}=\frac{N_{0} n_{0} \nu(0)}{2}+\left(N-N_{0}\right) n_{0} \nu(0)+\delta E_{0}+ \\
& +\frac{1}{2} \sum_{l=1,2, \ldots, \infty}\left(E_{b}\left(k_{l}\right)-K\left(k_{l}\right)-n_{0} \nu\left(k_{l}\right)\right) .
\end{aligned}
$$

We remark that the operators $\hat{\xi}_{l}^{+}, \hat{\xi}_{p}$ (33) (with replacements $\hat{b}_{2 j+1} \rightarrow \hat{\tilde{b}}_{2 j+1}, \hat{b}_{2 j+1}^{+} \rightarrow \hat{\tilde{b}}_{2 j+1}^{+}$or without them) satisfy the commutation relations (13) for Bose operators.

According to solution (61), (62), the system can be considered as a gas of noninteracting quasiparticles with the energy $E_{b}(k)$, and the number of quasiparticles can vary. Our solution differs from the Bogoliubov one by that the quasiparticles are standing waves rather than traveling ones, which is natural, and by the term $\delta E_{0}$. It is shown in Appendix B that $\delta E_{0}$ is negligible provided that the coupling is weak, and the interaction radius is less or comparable with the interatomic distance. Under these conditions, our solutions $E(k)$ and $E_{0}$ coincide with the Bogoliubov ones for a periodic system.

ISSN 2071-0194. Ukr. J. Phys. 2019. Vol. 64, No. 3 
We note that if the interaction radius tends to zero, then the energy levels $E_{b}(k)$ with $k=\pi / L, 2 \pi / L, \ldots$ coincide with the corresponding levels $[8,9]$ of a system of point bosons, which is described via the Bethe ansatz.

Since the excitations are standing waves, index $l$ in (61), (62) takes only positive values $(1,2,3, \ldots)$. As $k_{l}$ varies with the step $\Delta k=\pi / L$, the value of the sum $\sum_{l} f\left(k_{l}\right)$ for zero BCs is the same as for periodic BCs, for which $l= \pm 1, \pm 2, \pm 3, \ldots$ and $\triangle k=2 \pi / L$ [1]. Therefore, the values of thermodynamic quantities under zero and periodic BCs are identical (the same result was obtained for point bosons [8]).

We remark also that the replacement by a c-number was made twice (rough and fine tunings): first, we separated $d_{2 j+1}^{(0)}$ from $\hat{d}_{2 j+1}$ and, second, $\beta_{2 j+1}$ from $\hat{b}_{2 j+1}$. The latter is equivalent to the separation of an additional c-number $\beta_{2 j+1} e^{\epsilon_{0} t / i \hbar}$ from $\hat{d}_{2 j+1}$ : that is, $d_{2 j+1}=d_{2 j+1}^{(0)}+\beta_{2 j+1} e^{\epsilon_{0} t / i \hbar}$. If the $(2 j+1)$-state is macroscopically occupied, then $\left|\beta_{2 j+1}\right| \ll\left|d_{2 j+1}^{(0)}\right|$.

Note that, in the Hamiltonian, we neglected small corrections of the kind $\hat{a}_{j}^{3}, \hat{a}_{j}^{4}$, which leads to the absence of the interaction between quasiparticles. Therefore, the model describes only such states of the system, for which the interaction of quasiparticles is inessential, i.e., the states with a not large number of quasiparticles. The criterion of applicability of the method (see Section 5 below) imposes the stronger restriction on the number of quasiparticles.

It is also worth noting that, in the Bogoliubov method after the replacement $\hat{d}_{0} \rightarrow d_{0}$, the exact Hamiltonian (37)-(39) does not commute with the operator $\hat{N}=\Sigma_{j} \hat{d}_{j}^{+} \hat{d}_{j}$ of the total number of particles. Therefore, the number of particles $N$ is not conserved. Several close modifications of the Bogoliubov method, in which the number of particles is conserved, were proposed $[12,16,17,20]$. In our method, replacement (8) does not cause the violation of the equality $[\hat{H}, \hat{N}]=0$. However, for the diagonal Hamiltonian $(61)$, we have $[\hat{H}, \hat{N}] \neq 0$. The law of conservation of $N$ is broken due to the neglect of corrections of the orders $\vartheta^{3}$ and $\vartheta^{4}$ in the exact $\hat{H}$. It is necessary to modify the model so that the law of conservation of $N$ be satisfied. On the other hand, in order that $\langle\hat{\psi}(x, t)\rangle$ exist and be nonzero, one needs to break the invariance of the Hamiltonian relative to the transformation $\hat{\psi} \rightarrow e^{i \alpha} \hat{\psi}[13,14]$, which causes the violation of the law of conservation of $N$ (at least, formally).

\section{A Criterion of Applicability of the Method}

To find the condition of applicability of the method, we need to calculate the "anomalous" averages $\left.\left\langle\hat{d}_{l}^{+} \hat{d}_{j}\right\rangle\right|_{l \neq j}$ and $\langle\hat{\psi}(x, t)\rangle$. The symmetry-based reasoning $[13,14]$ implies that the law of conservation of the number of particles leads to $\langle\hat{\psi}(x, t)\rangle=0$. However, the Bogoliubov model gives a nonzero value of $\langle\hat{\psi}(x, t)\rangle$. This contradiction can be removed with the help of the introduction of a negligible correction with a certain structure into the Hamiltonian (the method of quasiaverages $[13,14])$. In this case, the law of conservation of the number of particles is formally broken, the average $\langle\hat{\psi}(x, t)\rangle$ can be nonzero, and the method of quasiaverages allows one to find it. However, in order to calculate $\langle\hat{\psi}(x, t)\rangle$, there is no need to use the method of quasiaverages. It is sufficient to use the quasiparticle representation [34] in formula (6). We express $\hat{d}_{j}^{+}$and $\hat{d}_{j}$ in the operator $\hat{A}$ in (6) through the operators $\hat{\xi}_{j}^{+}$and $\hat{\xi}_{j}$. Then we construct the collection $\left\{\Psi_{p}\left(x_{1}, \ldots, x_{N}\right)\right\}$ from the wave function of the ground state $\Psi_{0}$, the wave functions of states with one quasiparticle $\left(C_{l} \hat{\xi}_{l}^{+} \Psi_{0}\right)$, two quasiparticles $\left(C_{l j} \hat{\xi}_{l}^{+} \hat{\xi}_{j}^{+} \Psi_{0}\right)$, and so on $\left(C_{l}\right.$ and $C_{l j}$ are normalization factors). These functions are the eigenfunctions of Hamiltonian (61). The functions $\Psi_{p}$ of a Bose gas have the same structure [3]. It is known that the Schrödinger equation with given BCs has a set of solutions, which form a complete collection $\left\{\Psi_{p}\left(x_{1}, \ldots, x_{N}\right)\right\}$. It is clear that the lowest states in this collection should coincide with the aboveconstructed ones. The energy levels in (6) are described by the formula

$E_{p}=E_{0}+\sum_{l=1,2, \ldots, \infty} n_{l} E_{b}\left(k_{l}\right)$,

where the occupation numbers take values $n_{l}=$ $=0,1,2, \ldots, \infty$ for all $l$. In such way, we can find any averages. In this case, the system of interacting particles with a fixed $N$ is described as an ideal gas of quasiparticles, whose number varies.

Let us find the criterion of applicability of the method. The equations

$\sum_{j=1,2, \ldots, \infty} \hat{N}_{j}=\hat{N}, \quad \hat{N}_{j}=\hat{d}_{j}^{+} \hat{d}_{j}$ 
yield

$$
\sum_{j=1,2, \ldots, \infty} \frac{\left\langle\hat{d}_{j}^{+} \hat{d}_{j}\right\rangle}{N}=1,
$$

where $\left\langle\hat{d}_{j}^{+} \hat{d}_{j}\right\rangle=\left\langle\hat{N}_{j}\right\rangle$ is the average number of atoms in the state $\sqrt{2 / L} \cdot \sin \left(k_{j} x\right)$. For $j=2 l$, we have $\left\langle\hat{d}_{2 l}^{+} \hat{d}_{2 l}\right\rangle=\left\langle\hat{b}_{2 l}^{+} \hat{b}_{2 l}\right\rangle$. In order to determine $\left\langle\hat{b}_{2 l}^{+} \hat{b}_{2 l}\right\rangle$ by formula (6), we express $\hat{b}_{2 l}^{+}, \hat{b}_{2 l}$ in terms of $\hat{\xi}_{2 l}^{+}, \hat{\xi}_{2 l}$ according to $(32)$ and take into account that $\left\langle\hat{\xi}_{p}^{+}\right\rangle=$ $=\left\langle\hat{\xi}_{p}\right\rangle=0$. We obtain

$$
\begin{aligned}
& \left\langle\hat{b}_{2 l}^{+} \hat{b}_{2 l}\right\rangle=\frac{\left\langle\hat{n}_{2 l}\right\rangle+\left|\Lambda_{2 l}\right|^{2}\left(\left\langle\hat{n}_{2 l}\right\rangle+1\right)}{1-\left|\Lambda_{2 l}\right|^{2}}, \\
& \frac{\left|\Lambda_{p}\right|^{2}}{1-\left|\Lambda_{p}\right|^{2}}=\frac{\left(n_{0} \nu\left(k_{p}\right)\right)^{2}\left(2 E_{b}\left(k_{p}\right)\right)^{-1}}{E_{b}\left(k_{p}\right)+K\left(k_{p}\right)+n_{0} \nu\left(k_{p}\right)} .
\end{aligned}
$$

In the approximation of free quasiparticles, the average number of quasiparticles with the quasimomentum $k=k_{l}$ is determined by the Bose distribution $[34,39,40]$ :

$$
\left\langle\hat{n}_{l}\right\rangle=\left\langle\hat{\xi}_{l}^{+} \hat{\xi}_{l}\right\rangle=\left(e^{E_{b}\left(k_{l}\right) / k_{\mathrm{B}} T}-1\right)^{-1} .
$$

In this case, $\left.\left\langle\hat{n}_{l}\right\rangle\right|_{T=0}=0$. Formula (66) with $\left\langle\hat{n}_{2 l}\right\rangle=$ $=0$ follows also from $(7),(32)$.

With regard for (8) and (56), we obtain

$$
\begin{aligned}
& \left\langle\hat{d}_{2 j+1}^{+} \hat{d}_{2 j+1}\right\rangle=\left\langle\left(\hat{\tilde{b}}_{2 j+1}^{+}+\beta_{2 j+1}^{*}+f_{2 j+1}^{*}\right) \times\right. \\
& \left.\times\left(\hat{\tilde{b}}_{2 j+1}+\beta_{2 j+1}+f_{2 j+1}\right)\right\rangle .
\end{aligned}
$$

By expressing the operators $\hat{\tilde{b}}_{2 j+1}^{+}, \quad \hat{\tilde{b}}_{2 j+1}$ through $\hat{\xi}_{2 j+1}^{+}, \hat{\xi}_{2 j+1}$ according to $(32)$, we find

$$
\begin{aligned}
& \left\langle\hat{d}_{2 j+1}^{+} \hat{d}_{2 j+1}\right\rangle=G_{2 j+1}+\left\langle\hat{\tilde{b}}_{2 j+1}^{+} \hat{\tilde{b}}_{2 j+1}\right\rangle, \\
& G_{2 j+1}=\left(\beta_{2 j+1}^{*}+f_{2 j+1}^{*}\right)\left(\beta_{2 j+1}+f_{2 j+1}\right), \\
& \left\langle\hat{\tilde{b}}_{2 j+1}^{+} \hat{\tilde{b}}_{2 j+1}\right\rangle=\frac{\left\langle\hat{n}_{2 j+1}\right\rangle+\left|\Lambda_{2 j+1}\right|^{2}\left(\left\langle\hat{n}_{2 j+1}\right\rangle+1\right)}{1-\left|\Lambda_{2 j+1}\right|^{2}} .
\end{aligned}
$$

The above formulae yield

$$
\begin{aligned}
& 1-\frac{\tilde{N}_{0}}{N}=\frac{1}{N} \times \\
& \times \sum_{j=1,2, \ldots, \infty} \frac{\left(n_{0} \nu\left(k_{j}\right)\right)^{2}}{2 E_{b}\left(k_{j}\right)\left[E_{b}\left(k_{j}\right)+K\left(k_{j}\right)+n_{0} \nu\left(k_{j}\right)\right]}+ \\
& +\frac{1}{N} \sum_{j=1,2, \ldots, \infty} \frac{K\left(k_{j}\right)+n_{0} \nu\left(k_{j}\right)}{\left[e^{E_{b}\left(k_{j}\right) / k_{\mathrm{B}} T}-1\right] E_{b}\left(k_{j}\right)},
\end{aligned}
$$

where $\tilde{N}_{0}$ is the number of atoms in the effective condensate $\langle\hat{\psi}(x, t)\rangle$ :

$$
\begin{aligned}
& \tilde{N}_{0}=\int_{0}^{L}|\langle\hat{\psi}(x, t)\rangle|^{2} d x= \\
& =\sum_{j=0, \ldots, \infty}^{0}\left\langle\hat{d}_{2 j+1}^{+}(t)\right\rangle\left\langle\hat{d}_{2 j+1}(t)\right\rangle=\sum_{j=0, \ldots, \infty} G_{2 j+1} .
\end{aligned}
$$

Here, $\left\langle\hat{d}_{2 j+1}(t)\right\rangle=e^{\epsilon_{0} t / i \hbar}\left[f_{2 j+1}+\beta_{2 j+1}\right]$. According to the above analysis, the effective condensate is described by the formula

$$
\langle\hat{\psi}(x, t)\rangle=\sum_{j=0,1, \ldots, \infty}\left\langle\hat{d}_{2 j+1}(t)\right\rangle \sqrt{\frac{2}{L}} \sin \left(k_{2 j+1} x\right) .
$$

For simplicity, we consider the point potential $U\left(\left|x_{j}-x_{l}\right|\right)=2 c \delta\left(x_{j}-x_{l}\right)$ (i.e. $\left.\nu(k)=2 c\right)$ and set $n=\frac{N}{L}, \frac{K(k)}{n_{0} 2 c}=\frac{k^{2}}{2 n_{0} n \gamma}$, where $\gamma=\frac{2 m c}{\hbar^{2} n}$ coincides with $\gamma$ by Lieb-Liniger [5]. Then

$f_{j}+\beta_{j}=f_{j} \frac{4 \gamma n_{0} n}{k_{j}^{2}+4 \gamma n_{0} n}$

$G_{j}=\left|f_{j}\right|^{2} \frac{\left(4 \gamma n_{0} n\right)^{2}}{\left(k_{j}^{2}+4 \gamma n_{0} n\right)^{2}}=\frac{8 N_{0}}{\pi^{2} j^{2}} \frac{1}{\left[y_{j}^{2} / 4+1\right]^{2}}$,

$\tilde{N}_{0} \equiv \sum_{j=0,1, \ldots, \infty} G_{2 j+1}=N_{0}\left(1-\frac{1.5}{\pi \sqrt{\Gamma}}\right)$,

$\chi_{j} \equiv \frac{\left(n_{0} \nu\left(k_{j}\right)\right)^{2}}{2 E_{b}\left(k_{j}\right)\left[E_{b}\left(k_{j}\right)+K\left(k_{j}\right)+n_{0} \nu\left(k_{j}\right)\right]}=$

$=\frac{2}{\sqrt{y_{j}^{4}+4 y_{j}^{2}}\left(\sqrt{y_{j}^{4}+4 y_{j}^{2}}+y_{j}^{2}+2\right)}$,

$\omega_{j} \equiv \frac{K\left(k_{j}\right)+n_{0} \nu\left(k_{j}\right)}{\left[e^{E_{b}\left(k_{j}\right) / k_{\mathrm{B}} T}-1\right] E_{b}\left(k_{j}\right)}=$

$=\frac{y_{j}^{2}+2}{\left[e^{\sqrt{y_{j}^{4}+4 y_{j}^{2}} / \tilde{T}}-1\right] \sqrt{y_{j}^{4}+4 y_{j}^{2}}}$,

$\sum_{j=1,2, \ldots, \infty} \chi_{j}=q_{0}(\Gamma) \frac{\sqrt{\Gamma} \ln \Gamma}{4}$

$\sum_{j=1,2, \ldots, \infty} \omega_{j}=q_{T}(\Gamma, \tilde{T}) 0.82 \tilde{T} \Gamma$

where $y_{j}=\frac{k_{j}}{\sqrt{\gamma n n_{0}}}=\frac{j}{\sqrt{\Gamma}}, \tilde{T}=\frac{k_{\mathrm{B}} T}{c n_{0}}, \Gamma=\frac{\gamma N N_{0}}{\pi^{2}}$. The equality (78) holds at $\Gamma \gtrsim 1$.

Relations (73)-(82) yield

$1-\frac{\tilde{N}_{0}}{N}=\frac{q_{0}(\Gamma) \sqrt{\Gamma}}{4 N} \ln \Gamma+q_{T}(\Gamma, \tilde{T}) \frac{0.82 \tilde{T} \Gamma}{N}$,

ISSN 2071-0194. Ukr. J. Phys. 2019. Vol. 64, No. 3 
where $q_{0}(\Gamma) \approx 1$ at $\Gamma \gg 1$, and $q_{T} \approx 1$ at $\Gamma \gg 1$, $\tilde{T} \gg \Gamma^{-1 / 2}$. The condition $\tilde{T} \gg \Gamma^{-1 / 2}$ follows from $E_{b}\left(k_{1}\right) \ll k_{\mathrm{B}} T$ (nonzero temperature has a meaning, if the system is in the equilibrium state; the equilibrium is possible for a large number of quasiparticles; the last is satisfied for $\left.E_{b}\left(k_{1}\right) \ll k_{\mathrm{B}} T\right)$.

Under periodic BCs, the solution [1] yields formula (83) with the replacement $\tilde{N}_{0} \rightarrow N_{0}$ and with the parameters $q_{0} \approx 0.9, q_{T} \approx 0.5($ at $\Gamma \gg 1, \tilde{T} \gg$ $\left.\gg \Gamma^{-1 / 2}\right)$.

We have used the approximation $\hat{\vartheta} \ll\langle\hat{\psi}(x, t)\rangle$, which holds for $N-\tilde{N}_{0} \ll N$. The condition $N-$ $-\tilde{N}_{0} \ll N$ implies that almost all atoms are in the effective condensate $\langle\hat{\psi}(x, t)\rangle$. From relation (83), we obtain the criterion of applicability of the method:

$N-\tilde{N}_{0} \ll N \Leftrightarrow 0<\frac{\sqrt{\gamma}}{2 \pi} \ln \frac{N \sqrt{\gamma}}{\pi}+0.08 \gamma N \tilde{T} \ll 1$.

At $N=\infty$ and $T=0$, inequalities (84) are broken for any finite $\gamma$. From (78), (83), we get $q_{0} \approx 1$, $\tilde{N}_{0}=N_{0} \approx N\left(\frac{2 \pi}{\sqrt{\gamma} \ln N}\right)^{2} \ll N$. Such value of $\tilde{N}_{0}$ can be considered macroscopic at $\sqrt{\gamma} \lesssim 2 \pi$ (for the system with finite $N$, we consider the $j$-th state to be macroscopically occupied, if $\left\langle\hat{N}_{j}\right\rangle \gtrsim N / \Theta$, where $\left.\Theta=\ln ^{2} N\right)$. If $N=\infty$ and $T>0$, then (83) implies that $\tilde{N}_{0}$ is microscopic. It was shown in [33] that $\tilde{N}_{0}$ is close to the number of atoms in the unique condensate. Such results agree with the conclusion of the well-known works $[26,27]$ that the existence of a condensate in an infinite $1 \mathrm{D}$ system at $T>0$ is impossible.

At finite $N$ and $T=0$, inequalities (84) hold provided that $\gamma$ is small. At finite $N$ and $T>0$, relation (84) yields $\gamma N \tilde{T} \ll 12$, and the condition $\tilde{T} \gg \Gamma^{-1 / 2}$ gives $\tilde{T} \gg \pi /(\sqrt{\gamma} N)$. These inequalities are compatible at $\gamma<10^{-3}$. Additionally, the relation $\frac{\sqrt{\gamma}}{2 \pi} \ln \frac{N \sqrt{\gamma}}{\pi} \ll 1$ should hold. This shows that, in a finite system, criterion (84) holds even at $T>0$, if $\gamma<10^{-3}$ and the temperature is small: $\tilde{T} \ll \frac{12}{\gamma N}$, i.e., $k_{\mathrm{B}} T \ll \frac{6 \hbar^{2} n^{2}}{m N}$. Interestingly, the last inequality contains no interaction constant c. At first sight, the condition $k_{\mathrm{B}} T \ll \frac{6 \hbar^{2} n^{2}}{m N}$ differs radically from the criterion of Bose condensation for the ideal Bose gas in a $1 \mathrm{D}$ trap: $k_{\mathrm{B}} T \lesssim \frac{N \hbar \omega}{\ln (2 N)}$ [41], where $\omega$ is the trap frequency. But this is not the case. Zero BCs are similar to a trap with the frequency $\hbar \omega=\frac{\hbar^{2} k_{\min }^{2}}{2 m}$, where $k_{\min }=\frac{\pi}{L}$. Therefore, the ISSN 2071-0194. Ukr. J. Phys. 2019. Vol. 64, No. 3 criterion $k_{\mathrm{B}} T \lesssim \frac{N \hbar \omega}{\ln (2 N)}$ leads to $k_{\mathrm{B}} T \lesssim \frac{\pi^{2} \hbar^{2} n^{2}}{2 m N \ln (2 N)}$, which is close to $k_{\mathrm{B}} T \ll \frac{6 \hbar^{2} n^{2}}{m N}$.

Thus, our method can be used for the description of a finite system at low temperatures and with weak coupling.

\section{Single-Particle Density Matrix and the Quasicondensate}

For the completeness, we now find the density matrix:

$F_{1}\left(x, x^{\prime}\right)=\left\langle\hat{\psi}^{+}\left(x^{\prime}, t\right) \hat{\psi}(x, t)\right\rangle=$

$=\sum_{j_{1} j_{2}=1,2, \ldots, \infty} g_{2 j_{1}-1}^{*}\left(x^{\prime}\right) g_{2 j_{2}-1}(x) \times$

$\times\left[d_{2 j_{1}-1}^{(0) *} d_{2 j_{2}-1}^{(0)}+d_{2 j_{1}-1}^{(0) *}\left\langle\hat{a}_{2 j_{2}-1}\right\rangle+\right.$

$\left.+d_{2 j_{2}-1}^{(0)}\left\langle\hat{a}_{2 j_{1}-1}^{+}\right\rangle+\left\langle\hat{a}_{2 j_{1}-1}^{+} \hat{a}_{2 j_{2}-1}\right\rangle\right]+$

$+\sum_{l_{1} l_{2}=1,2, \ldots, \infty} g_{2 l_{1}}^{*}\left(x^{\prime}\right) g_{2 l_{2}}(x)\left\langle\hat{a}_{2 l_{1}}^{+} \hat{a}_{2 l_{2}}\right\rangle+$

$+\sum_{j_{1} l_{2}=1,2, \ldots, \infty} g_{2 j_{1}-1}^{*}\left(x^{\prime}\right) g_{2 l_{2}}(x) \times$

$\times\left[d_{2 j_{1}-1}^{(0) *}\left\langle\hat{a}_{2 l_{2}}\right\rangle+\left\langle\hat{a}_{2 j_{1}-1}^{+} \hat{a}_{2 l_{2}}\right\rangle\right]+$

$+\sum_{l_{1} j_{2}=1,2, \ldots, \infty} g_{2 l_{1}}^{*}\left(x^{\prime}\right) g_{2 j_{2}-1}(x) \times$

$\times\left[d_{2 j_{2}-1}^{(0)}\left\langle\hat{a}_{2 l_{1}}^{+}\right\rangle+\left\langle\hat{a}_{2 l_{1}}^{+} \hat{a}_{2 j_{2}-1}\right\rangle\right]$.

We take into account that $\hat{a}_{p}(t)=e^{\epsilon_{0} t / i \hbar} \hat{b}_{p}, \hat{b}_{2 j-1}=$ $=\hat{\tilde{b}}_{2 j-1}+\beta_{2 j-1}, d_{2 j-1}^{(0)}=e^{\epsilon_{0} t / i \hbar} f_{2 j-1}$. Let us express the operators $\hat{\tilde{b}}_{2 j-1}, \hat{b}_{2 j}$ in terms of $\hat{\xi}_{p}^{+}, \hat{\xi}_{p}$ with the help of relations $(32)$. We can verify that $\left\langle\hat{b}_{2 j}\right\rangle=$ $=\left\langle\hat{\tilde{b}}_{2 j-1}\right\rangle=0,\left\langle\hat{b}_{2 j}^{+}\right\rangle=\left\langle\hat{\tilde{b}}_{2 j-1}^{+}\right\rangle=0,\left\langle\hat{\tilde{b}}_{2 j_{1}-1}^{+} \hat{\tilde{\tilde{b}}}_{2 j_{2}-1}\right\rangle=$ $=\delta_{j_{1}, j_{2}}\left\langle\hat{\tilde{b}}_{2 j_{1}-1}^{+} \hat{\tilde{b}}_{2 j_{1}-1}\right\rangle,\left\langle\hat{b}_{2 l_{1}}^{+} \hat{b}_{2 l_{2}}\right\rangle=\delta_{l_{1}, l_{2}}\left\langle\hat{b}_{2 l_{1}}^{+} \hat{b}_{2 l_{1}}\right\rangle$, $\left\langle\hat{\tilde{b}}_{2 j_{1}-1}^{+} \hat{b}_{2 l_{2}}\right\rangle=\left\langle\hat{b}_{2 l_{1}}^{+} \hat{\tilde{b}}_{2 j_{2}-1}\right\rangle=0$. Then relation $(85)$ yields

$F_{1}\left(x, x^{\prime}\right)=\left\langle\hat{\psi}\left(x^{\prime}, t\right)\right\rangle^{*}\langle\hat{\psi}(x, t)\rangle+$

$+\sum_{l=1,2, \ldots, \infty} g_{2 l}^{*}\left(x^{\prime}\right) g_{2 l}(x)\left\langle\hat{b}_{2 l}^{+} \hat{b}_{2 l}\right\rangle+$

$+\sum_{j=1,2, \ldots, \infty} g_{2 j-1}^{*}\left(x^{\prime}\right) g_{2 j-1}(x)\left\langle\hat{\tilde{b}}_{2 j-1}^{+} \hat{\tilde{b}}_{2 j-1}\right\rangle$. 


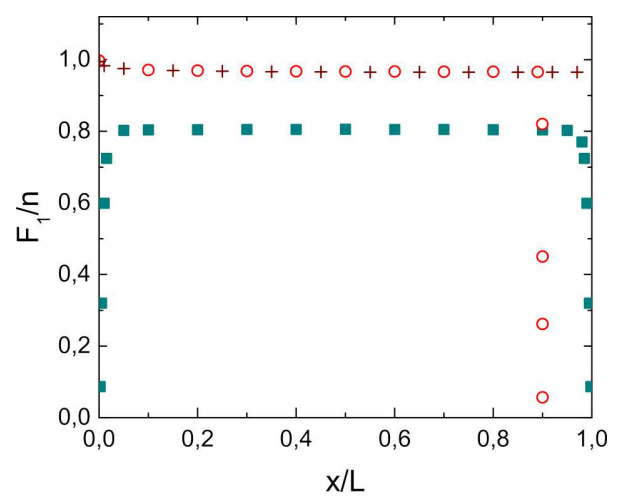

Concentration $n(x) / n=F_{1}(x, x) / n$ (squares, $N=10^{4}, \gamma=$ $=0.0001)$ and the density matrix $F_{1}\left(x_{1}, x_{1}+x\right) / n$ (open circles, $\left.x_{1}=0.1 L, N=10^{5}, \Gamma=10^{6}, \gamma \approx 0.00102\right)$ under zero BCs determined numerically by formulae (87), (88) at $T=0$. Crosses show solution (90) for the density matrix under periodic BCs. In order to separate the curves, the values of the concentration $n(x)$ are multiplied by 0.8

With regard for the formula $g_{l}(x)=\sqrt{2 / L} \sin \left(k_{l} x\right)$ and relations $(66),(67),(68),(72)$, for the point interaction $(\nu(k)=2 c)$, relation (86) yields

$$
\begin{aligned}
& F_{1}\left(x, x^{\prime}\right)=f_{0}^{*}\left(x^{\prime}\right) f_{0}(x)+ \\
& +\frac{2}{L} \sum_{l=1,2, \ldots, \infty} \frac{\sin \left(k_{l} x^{\prime}\right) \sin \left(k_{l} x\right)}{\sqrt{y_{l}^{4}+4 y_{l}^{2}}} \times \\
& \times\left(\frac{2}{\sqrt{y_{l}^{4}+4 y_{l}^{2}}+y_{l}^{2}+2}+\frac{y_{l}^{2}+2}{e^{\frac{\sqrt{y_{l}^{4}+4 y_{l}^{2}}}{\tilde{T}}}-1}\right), \\
& f_{0}(x)=\frac{4 \sqrt{n_{0}}}{\pi} \sum_{j=1,2, \ldots, \infty} \frac{\sin \left(k_{2 j-1} x\right)}{2 j-1} \frac{4}{y_{2 j-1}^{2}+4} \text {, }
\end{aligned}
$$

where $y_{l}=l / \sqrt{\Gamma}$. The total concentration of the gas is given by the formula

$n(x)=F_{1}(x, x)$.

In Figure, we show the concentration $n(x)$ and the density matrix $F_{1}\left(x_{1}, x_{1}+x\right)$ (as a function of $x$ ) determined numerically from (87)-(89) for $T=0$. In this case, we obtained $N_{0}$ from (78), (83). As is seen, the concentration is constant inside the system, decreases by approaching the boundary, and turns to zero on the boundary. The width of a band near the boundary, where the concentration varies, is $\delta L \approx L / \sqrt{\Gamma}=\pi / \sqrt{\gamma n n_{0}}$. The density matrix $F_{1}\left(x_{1}, x_{1}+x\right)$ depends on $x$ and is almost independent of $x_{1}$, if the point $x_{1}$ is at a distance $\gtrsim \delta L$ from the boundary. It is seen from Figure that $\left.F_{1}\left(x_{1}, x_{1}+x\right)\right|_{T=0}(87)$ is very close to the solution for a periodic system at $T=0$, which reads [40,42-49]

$\left.F_{1}\left(x_{1}, x_{1}+x\right)\right|_{|x|} \gtrsim \delta L=n\left(\frac{\delta L f_{2}(|x|)}{|x|}\right)^{\sqrt{\gamma} / 2 \pi}$.

The available literature gives several close values for $f_{2}(x): 1 / \pi[40,43-45], 0.33$ [46-48], and $\frac{1}{3}[0.98+$ $\left.+2(x / L)^{2}\right]$ [42]. In Figure, we present function (90) with $f_{2}(x)=\frac{1}{3}\left[0.98+2(x / L)^{2}\right]$. Such $f_{2}(x)$ leads to a slightly better agreement than $f_{2}(x)=1 / \pi ; 0.33$. It is seen that, as $x$ varies from 0 to $L-x_{1}$, the function $F_{1}\left(x_{1}, x_{1}+x\right)$ is changed only by $3 \%$ at $\gamma \approx 0.00102$, $|x| \gtrsim \delta L$. For $\gamma=0.0001$, the function $F_{1}\left(x_{1}, x_{1}+x\right)$ varies only by $0.5 \%$, as $x$ increases. If $F_{1}\left(x_{1}, x_{1}+x\right)$ decreases (as the function of $x$ ) by a power law, the term "quasicondensate" [50] is usually used instead of "condensate". But our solution $F_{1}\left(x_{1}, x_{1}+x\right)$ decreases, as $x$ increases, very slowly for any parameters $\gamma$ and $N$ satisfying criterion (84). That is, we have the true condensate for such parameters. Such regime was found previously in [42]. For the applicability of our method, it is insignificant whether $\langle\hat{\psi}(x, t)\rangle$ corresponds to the condensate or quasicondensate. It is important that the system be finite.

Note that the curve $F_{1}\left(x_{1}, x_{1}+x\right)$ (87) (circles in Figure) drops to zero at $x=0.9 L$. This is due to the fact that Figure is plotted for $x_{1}=0.1 L$. So, for $x=0.9 L$, the coordinate $x_{2}$ turns out to be on the boundary: $x_{2} \equiv x_{1}+x=L$.

Our analysis shows that, under zero BCs, the power law (90) holds for all $x_{1} \in[\delta L, L-\delta L], x \in[\delta L, L-$ $\left.-x_{1}-\delta L\right]$. In works by Cazalilla [10,11], $F_{1}\left(x, x^{\prime}\right)$ was also calculated for a $1 \mathrm{D}$ system of interacting bosons with zero BCs. According to those results, $F_{1}\left(x, x^{\prime}\right)$ decreases by a power law, as $\left|x-x^{\prime}\right|$ increases, only at the points near the vessel center. The deviation from a power law for the remaining points is related, apparently, to the harmonic-fluid approximation used in $[10,11]$.

We remark that the function $\left.F_{1}(0, x)\right|_{T=0}$ was also calculated for a periodic system of point bosons at intermediate values of $\gamma$ and finite $N[51,52]$.

By neglecting the terms $\hat{\vartheta}$ and $\delta \psi_{0}$ in the formula $\hat{\psi}=\psi_{0}(x, t)+\delta \psi_{0}(x, t)+\hat{\vartheta}(x, t)$, we obtain

$F_{1}\left(x, x^{\prime}\right)=\psi_{0}^{*}\left(x^{\prime}, t\right) \psi_{0}(x, t)=$

$=\left[\begin{array}{ll}n_{0}, & \left.\text { if } x, x^{\prime} \in\right] 0, L[ \\ 0, & \text { if } x=0 ; L \text { or } x^{\prime}=0 ; L .\end{array}\right.$

ISSN 2071-0194. Ukr. J. Phys. 2019. Vol. 64, No. 3 
In this case, we lost both the smooth decrease in $F_{1}$ near the boundaries and the power decrease by law (90).

Using formulae (87) and (88), we have found numerically the density matrix for $T>0$. At $\delta L \lesssim x_{1} \lesssim L-\delta L, 10 \delta L \lesssim x \lesssim L-10 \delta L, \frac{\pi}{\sqrt{\gamma} N} \ll$ $\ll \tilde{T} \lesssim \frac{4}{\gamma N}$, the solution takes the form

$\left.F_{1}\left(x_{1}, x_{1}+x\right)\right|_{T>0} \approx C_{T}\left(x_{1}\right) n e^{-0.5 \gamma n \tilde{T} x x_{1} L^{-1}}$,

where $C_{T}\left(x_{1}\right)$ is the normalization factor depending on $x_{1}$. If criterion $(84)(\gamma N \tilde{T} \lesssim 1)$ is satisfied, the function $F_{1}\left(x_{1}, x_{1}+x\right)(92)$ decreases weakly, as $x$ increases. This corresponds to the true condensate. At $0<\tilde{T} \lesssim \frac{\pi}{\sqrt{\gamma} N}$, the solution is intermediate between (90) and (92). We did not find it, since such temperatures are not quite physical (at such $\tilde{T}$, the system contains only several quasiparticles, and the thermal equilibrium is impossible). We note that solution (92) differs significantly from the solution for a periodic system, which is independent of $x_{1}[43,46,48]$ :

$\left.F_{1}\left(x_{1}, x_{1}+x\right)\right|_{T>0} \approx$
$\approx n\left(\frac{\delta L f_{2}(|x|)}{|x|}\right)^{\sqrt{\gamma} / 2 \pi} e^{-0.25 \gamma n_{0} \tilde{T}|x|}$.

Let us write the wave function of the effective condensate in the form $\langle\hat{\psi}(x, t)\rangle=\sqrt{n_{c}(x)} e^{i \alpha(x, t)}$, $n_{c}(x)=f_{0}^{2}(x)$. The numerical solution indicates that, at $\gamma \ll 1, T=0$, the condensate concentration $n_{c}(x)$ is very close to the total concentration $n(x): \quad n_{c}(x)=(1-|\kappa(x)|) n(x)$, where $|\kappa(x)| \ll 1$. In particular, for $N=10^{4}, \gamma=0.0001$, we have $\kappa(x)=0.001-0.007$.

We remarked above that the concentration $\left.n(x)\right|_{T=0}$ is nonuniform near the boundaries in the layer of thickness $\delta L \approx \pi / \sqrt{\gamma n n_{0}}$. For $\gamma \lesssim N^{-2}$, we obtain $\delta L \gtrsim L$ : the concentration is nonuniform in the whole system. This corresponds to the regime of almost free particles. Our model does not work for it, since the approximation $\hat{\vartheta} \ll\langle\hat{\psi}(x, t)\rangle$ is violated. At $\gamma \lesssim N^{-2}$, the correction $\delta E_{0}(60)$ becomes large (see Appendix B). The value of $E_{0}$ for this regime was found for point bosons described via the Bethe ansatz $[8,53]$.

\section{Summary}

We have generalized the Bogoliubov method and have constructed a description of low-lying levels of a one-dimensional system of weakly interacting bosons under zero boundary conditions. Two points are significant for the method: (i) the representation $\hat{\psi}(x, t)=\langle\hat{\psi}(x, t)\rangle+\hat{\vartheta}(x, t)$ and (ii) the separation of the principal part in integrals. We emphasize that the method requires the existence of the order parameter $\langle\hat{\psi}(x, t)\rangle$, rather than a condensate (determined on the basis of a diagonal expansion of the density matrix). Sometimes, the average $\langle\hat{\psi}(x, t)\rangle$ coincides with a condensate [1]; sometimes, $\langle\hat{\psi}(x, t)\rangle$ is only close to the condensate [33]. Apparently, it is possible that the condensate (quasicondensate) is absent, but $\langle\hat{\psi}(x, t)\rangle \neq 0$, and the method is valid. Though we were not faced with such case yet.

In the subsequent work [33] we diagonalized the density matrix (87). It turned out that the average $\langle\hat{\psi}(x, t)\rangle$ does not quite coincide with the true quasicondensate defined on the basis of expansion (5). Moreover, the quasicondensate is fragmented, and its structure depends on the boundary conditions.

In Appendix A, we argue that the Bogoliubov quasiparticles are collective excitations, though they look as one-particle excitations.

It is important that our solutions for $E_{0}$ and $E(k)$ coincide with those obtained by the exactly solvable approach, based on the Bethe ansatz [79]. In addition, our solution for the density matrix $\left.F_{1}\left(x_{1}, x_{2}\right)\right|_{T=0}$ coincides with the solution obtained within other methods for periodic BCs [40, 42-49]. This clearly shows that the Bogoliubov approximation is quite accurate for a finite 1D Bose system with weak coupling. Interestingly, for the point interaction, the Bogoliubov solutions $E_{0}$ and $E(k)$ are approximately valid also at $\gamma \sim 1$ even in the limit $N \rightarrow \infty[5,8,9]$, which contradicts criterion (84). This means that the region of applicability of the Bogoliubov solutions is much wider than the region of applicability of the Bogoliubov method.

We have found that the bulk properties $\left(E_{0}\right.$ and $E(k))$ of the system with boundaries are the same as for a periodic system. This is understandable in view of the definition

$E_{0}=\int d x_{1} \ldots d x_{N} \Psi_{0}^{*}\left[-\frac{\hbar^{2}}{2 m} \sum_{j=1}^{N} \frac{\partial^{2}}{\partial x_{j}^{2}}+\right.$
$\left.+\sum_{j<l} U\left(\left|x_{j}-x_{l}\right|\right)\right] \Psi_{0}$. 
In order that $E_{0}$ depend on the boundaries, it is necessary that the roughness of a landscape of the wave function $\Psi_{0}\left(x_{1}, \ldots, x_{N}\right)$ or the probability of overlapping of two adjacent atoms be dependent on the boundaries. However, both these properties have a local nature and, apparently, should not depend on the remote boundaries. If such dependence would exist, we would have a nontrivial effect. In order that such effect be, it is necessary that a solution with a substantially smaller energy than $E_{0}$ obtained above (see Eq. (62)) exist. However, our ground state is characterized by the uniform effective condensate $\langle\hat{\psi}(x, t)\rangle$, which contains almost all atoms. We see no state, which would possess a smaller energy. Therefore, we may conclude with a high degree of confidence that the boundaries do not affect the bulk properties of a system of weakly interacting bosons. Though, it would be of high interest, if such influence would exist.

The author is grateful to Yu. Shtanov for the valuable discussion. The present work is partially supported by the National Academy of Sciences of Ukraine (project No. 0116U003191).

\section{APPENDIX A.}

\section{Remarks on the Bogoliubov method}

According to the Bogoliubov transformations (33), the operator of creation of a quasiparticle $\hat{\xi}_{p}^{+}$is the sum of the operators of creation $\left(\hat{b}_{p}^{+}\right)$and annihilation $\left(\hat{b}_{p}\right)$ of one particle. From whence, many authors made conclusion about the single-particle nature of Bogoliubov quasiparticles. However, the solutions by Feynman [2] and by Bogoliubov-Zubarev [3] clearly show that a quasiparticle is a collective oscillation of the whole gas (condensate and above-condensate atoms). Let us consider this point.

First, one-particle excitations are impossible in the onedimensional case, since the moving atom will necessarily collide with the neighbor atom [54]. But the Bogoliubov solutions in $1 \mathrm{D}, 2 \mathrm{D}$, and $3 \mathrm{D}$ cases are similar. Hence, the Bogoliubov quasiparticles in $1 \mathrm{D}, 2 \mathrm{D}$, and $3 \mathrm{D}$ should be collective and should coincide with quasiparticles in [2,3]. The solutions for $E_{0}$ and $E(k)$ obtained in the Bogoliubov [1] and collective [3] approaches coincide.

The method of secondary quantization is based on that the $N$-particle Bose-symmetric wave function of the system can be presented as the expansion [34]

$$
\begin{aligned}
& \Psi\left(x_{1}, \ldots, x_{N}\right)=\sum_{\left\{n_{f}\right\}} C\left(n_{f_{1}}, \ldots, n_{f_{N}}\right) \psi_{\left\{n_{f}\right\}}\left(x_{1}, \ldots, x_{N}\right), \\
& \psi_{\left\{n_{f}\right\}}\left(x_{1}, \ldots, x_{N}\right)=\tilde{c}_{\left\{n_{f_{j}}\right\}} \sum_{P} P \varphi_{f_{1}}\left(x_{1}\right) \ldots \varphi_{f_{N}}\left(x_{N}\right) .
\end{aligned}
$$

Here, $\left\{n_{f}\right\}=\left(n_{f_{1}}, \ldots, n_{f_{N}}\right), n_{f_{1}}+\ldots+n_{f_{N}}=N$, the functions $\varphi_{l}(x)$ form a complete orthonormalized collection, the number $n_{f_{j}}=0,1,2, \ldots$ indicates the number of identical functions $\varphi_{f_{j}}(x)$ in expansion (96) (each index $f_{j}$ runs the same values as $l$ ), and $P$ means all possible permutations. Based on formulae (95), (96), and

$\hat{\psi}(x)=\sum_{l} \hat{b}_{l} \varphi_{l}(x)$

one can obtain the basic formulae of the method: (14) and (37)-(39) [34]. We now pass from $\hat{b}_{p}^{+}, \hat{b}_{p}$ to the operators of creation and annihilation of quasiparticles $\hat{\xi}_{p}^{+}, \hat{\xi}_{p}$. In this case, we consider the basis functions $\tilde{\varphi}_{l}(x)$ from the equality $\hat{\psi}(x)=\sum_{l} \hat{\xi}_{l} \tilde{\varphi}_{l}(x)$ instead of the functions $\varphi_{l}(x)$. Each eigenfunction $\Psi_{\left\{n_{f}\right\}}$ of the Hamiltonian can be represented

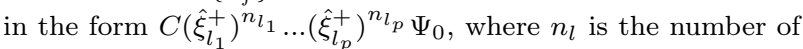
quasiparticles corresponding to index $l$. These numbers $n_{l}$ coincide with the numbers $n_{l}$ in relation (63) and do not coincide with the numbers $n_{l}$ in expansion (96) executed in the singleparticle basis $\left\{\tilde{\varphi}_{l}(x)\right\}$ (in the case of coincidence, we would get the wave function of the ground state $\Psi_{0}\left(x_{1}, \ldots, x_{N}\right)=$ const, since $n_{f_{1}}=n_{f_{2}}=\ldots=n_{f_{N}}=0$ for the state without quasiparticles; but the function $\Psi_{0}\left(x_{1}, \ldots, x_{N}\right)=$ const is not a solution of the Schrödinger equation with an interaction; moreover, the requirement $n_{f_{1}}+\ldots+n_{f_{N}}=N$ is violated). This means that a quasiparticle is not a single-particle structure, despite the single-particle form of formulae (33). We see also that the Bogoliubov method does not allow one to find the explicit form of the eigenfunctions $\Psi_{\left\{n_{f}\right\}}\left(x_{1}, \ldots, x_{N}\right)$. However, the diagonalization of the Hamiltonian enables one to find all observable parameters of the system: lowest energy levels $E_{j}$ (63), concentration $n(x)$ (89), correlation functions, and thermodynamic parameters.

The experiment [55] seems to be consistent with the singleparticle interpretation of formulae (33), according to which $\hat{b}_{p}^{+}$creates one atom. Note that the formula $\hat{b}_{p}^{+}\left|N_{p}=j\right\rangle=$ $=\sqrt{j+1}\left|N_{p}=j+1\right\rangle$ used in [55] is valid for the ideal gas with a variable number of particles and gives zero anomalous averages $\left\langle\hat{b}_{\mathbf{k}}^{+} \hat{b}_{-\mathbf{k}}^{+}\right\rangle,\left\langle\hat{b}_{\mathbf{k}} \hat{b}_{-\mathbf{k}}\right\rangle$. But, in the Bogoliubov model, the averages $\left\langle\hat{b}_{\mathbf{k}}^{+} \hat{b}_{-\mathbf{k}}^{+}\right\rangle,\left\langle\hat{b}_{\mathbf{k}} \hat{b}_{-\mathbf{k}}\right\rangle$ are nonzero and play the important role $[1,33]$. In the Bogoliubov approach, the state with one quasiparticle is described by the wave function $\hat{\xi}_{p}^{+} \Psi_{0}=$ $=\left(1-\left|\Lambda_{p}\right|^{2}\right)^{-1 / 2}\left(\hat{b}_{p}^{+}-\Lambda_{p}^{*} \hat{b}_{p}\right) \Psi_{0}$, which is a superposition of the state with $N+1$ particles and the state with $N-1$ particles ( $\Psi_{0}$ is a state with $N$ particles). In contrast, in the models $[2,3]$, the wave functions for the ground state and states with one quasiparticle are eigenfunctions of the Schrödinger equation with the same fixed number of particles $N$. We assume that such contradiction is due to the limitation of the language of operators $\hat{b}_{p}^{+}, \hat{b}_{p}$ : some properties expressed in this language become distorted. It arose, apparently, because the method of secondary quantization is based on a formalism with a variable number of particles $N$, but it is used for the calculation of the energy eigenvalues of the Schrödinger equation with fixed $N$. Despite this difficulty, the Bogoliubov method gives 
results for the observable quantities in agreement with other approaches.

We note that the many-particle nature of excitations in the Bogoliubov model is hidden [55] in the values of the coefficients $u_{p}=\left(1-\left|\Lambda_{p}\right|^{2}\right)^{-1 / 2}$ and $v_{p}=-\Lambda_{p}^{*}\left(1-\left|\Lambda_{p}\right|^{2}\right)^{-1 / 2}$ from (33): at a small quasimomentum $k=\pi p / L$, we have $u_{p} \approx\left|v_{p}\right| \gg 1$ (excitation involves many atoms); for large $k$, we get $u_{p} \simeq 1$, $v_{p} \simeq 0$ (excitation is mainly connected with one atom).

We remark also that, in the calculation of the average $\langle\hat{A}(x, t)\rangle$, one needs to use the canonical ensemble (for a system with fixed $N$ ) or the grand canonical ensemble (for a system with variable $N$ ). In a system with zero BCs, the number of particles must be conserved. Therefore, in formula (6), we based ourselves on the canonical ensemble. Bogoliubov [1] used the canonical ensemble as well (it is clearly seen from the analysis in [34]).

\section{APPENDIX B.}

\section{Corrections $S_{j}$ and $\delta E_{0}$}

1) Below, we list the formulae and estimates for the functions $S_{j}(x)$ from Sections 3 and 4 . The numerical calculations are carried out for the potential

$U\left(\left|x-x^{\prime}\right|\right)=\frac{c_{0}}{a} e^{-\left|x-x^{\prime}\right| / a}$,

which is characterized by the interaction radius $a$ and the Fourier transform $\nu\left(k_{j}\right)=\frac{2 c_{0}\left(1-e^{-L / a} \cos (\pi j)\right)}{1+\left(k_{j} a\right)^{2}}, k_{j}=\pi j / L$. We consider the interaction radius to be small $(a \ll L)$ and set $e^{-L / a}=0$. For each function $S_{j}$, we give the exact formula and a numerical estimate made for potential (98). The results are the following:

$$
\begin{aligned}
& S_{1}(x)=\frac{2}{L} \sum_{j=1,3,5, \ldots, \infty} \frac{\sin \left(k_{j} x\right)}{k_{j}}\left(\frac{\nu\left(k_{j}\right)}{\nu(0)}-1\right) \approx \\
& \approx \frac{1}{\pi} \int_{0}^{\infty} d y \frac{\sin (y x / a)}{y}\left(\frac{\nu(y / a)}{\nu(0)}-1\right) .
\end{aligned}
$$

Here, for potential (98), we have $S_{1}(x=0, L)=-0.5$ on the boundaries. As the distance from boundaries increases, $\left|S_{1}\right|$ decreases. For $x \in] 5 a, L-5 a$ [, we have $\left|S_{1}\right| \lesssim a / L$. That is, the correction $S_{1}$ is not small near the boundaries. Therefore, solution (22) of Eq. (15) is incorrect near the boundaries. We neglected this fact in Section 3, since the inaccuracy of the solution near the boundaries should not affect the bulk properties of the system. We have also

$$
\begin{aligned}
& S_{2}(l)=S_{22}(l)=\sum_{j=1,3,5, \ldots, \infty} \frac{2}{\pi^{2}}\left(\frac{\nu\left(k_{j}\right)}{\nu\left(k_{2 l}\right)}-1\right) \times \\
& \times\left(\frac{1}{2 l-j}+\frac{1}{2 l+j}\right)^{2}, \\
& 0<S_{2} \lesssim 80 l^{2}\left(\frac{a}{L}\right)^{3}, \\
& \left.S_{3}\left(l_{1}, l_{2}\right)\right|_{l_{1} \neq l_{2}}=S_{24}\left(l_{1}, l_{2}\right)=\frac{2}{\pi^{2}} \sum_{j=1,3, \ldots, \infty} \nu\left(k_{j}\right) \times \\
& \times\left(\frac{1}{2 l_{1}-j}+\frac{1}{2 l_{1}+j}\right)\left(\frac{1}{2 l_{2}-j}+\frac{1}{2 l_{2}+j}\right),
\end{aligned}
$$

ISSN 2071-0194. Ukr. J. Phys. 2019. Vol. 64, No. 3

$$
\begin{aligned}
& 0<S_{3} \lesssim \frac{3}{\pi^{2}} c_{0} l_{1} l_{2}\left(\frac{8 a}{L}\right)^{3}, \\
& S_{4}(j)=S_{23}(j)=\sum_{l=0,1, \ldots, \infty} \frac{\nu\left(k_{2 l}\right)-\nu\left(k_{2 j+1}\right)}{\nu\left(k_{2 j+1}\right)} \times \\
& \times \frac{2 q(l)}{\pi^{2}}\left(\frac{1}{2 j+1-2 l}+\frac{1}{2 j+1+2 l}\right)^{2}=4 \frac{a^{2}}{L^{2}} \times \\
& \times\left(1+\sum_{l=1,2, \ldots} \frac{2}{1+(2 \pi l a / L)^{2}} \frac{1}{1-(2 l /(2 j+1))^{2}}\right), \\
& 0<S_{4} \lesssim 20(2 j+1)^{2}\left(\frac{a}{L}\right)^{3}, \\
& \left.S_{5}\left(j_{1}, j_{2}\right)\right|_{j_{1} \neq j_{2}}=S_{25}\left(j_{1}, j_{2}\right)= \\
& =\frac{2}{\pi^{2}} \sum_{l=0,1,2, \ldots, \infty} \nu\left(k_{2 l}\right) q(l) \times \\
& \times\left(\frac{1}{2 j_{1}+1-2 l}+\frac{1}{2 j_{1}+1+2 l}\right) \times \\
& \times\left(\frac{1}{2 j_{2}+1-2 l}+\frac{1}{2 j_{2}+1+2 l}\right), \\
& 0<S_{5} \lesssim \frac{6}{\pi^{2}} c_{0}\left(2 j_{1}+1\right)\left(2 j_{2}+1\right)\left(\frac{4 a}{L}\right)^{3}, \\
& S_{21}=\frac{4}{\pi^{2}} \sum_{j=1,3,5, \ldots, \infty} \frac{\nu\left(k_{j}\right)-\nu(0)}{\nu(0) j^{2}} \simeq-\frac{a}{L}, \\
& \tilde{S}_{26}(l)=\frac{2}{\pi^{2}} \sum_{j=1,3,5, \ldots, \infty} \frac{\nu\left(k_{j}\right)}{j \nu\left(k_{2 l}\right)} \times \\
& \times\left(\frac{2}{j}-\frac{1}{j-4 l}-\frac{1}{j+4 l}\right)=\frac{\nu(0)}{2 \nu\left(k_{2 l}\right)}-S_{26}(l), \\
& 40 l^{2}\left(\frac{a}{L}\right)^{3} \lesssim S_{26}(l) \lesssim 160 l^{2}\left(\frac{a}{L}\right)^{3}, \\
& \tilde{S}_{27}(j)=\frac{2}{\pi^{2}} \sum_{j_{0}=1,3,5, \ldots, \infty} \frac{\nu\left(k_{j_{0}}\right)}{j_{0} \nu\left(k_{2 j+1}\right)} \times \\
& \times\left(\frac{2}{j_{0}}-\frac{1}{j_{0}-4 j-2}-\frac{1}{j_{0}+4 j+2}\right)= \\
& =\frac{\nu(0)}{2 \nu\left(k_{2 j+1}\right)}-S_{27}(j), \\
& (2 j+1)^{2}\left(\frac{a}{L}\right)^{3} \lesssim 0.1 S_{27}(j) \lesssim(4 j+2)^{2}\left(\frac{a}{L}\right)^{3}, \\
& S_{28}\left(l_{1}, l_{2}\right)=\frac{2}{\pi^{2}} \sum_{j=1,3,5, \ldots, \infty} \frac{\nu\left(k_{j}\right)}{j} \times \\
& \times\left(\frac{1}{j-2 l_{1}+2 l_{2}}+\frac{1}{j+2 l_{1}-2 l_{2}}-\right. \\
& \left.-\frac{1}{j-2 l_{1}-2 l_{2}}-\frac{1}{j+2 l_{1}+2 l_{2}}\right) \text {, } \\
& -320 c_{0} l_{1} l_{2}\left(\frac{a}{L}\right)^{3} \lesssim S_{28}<0 \text {, } \\
& S_{29}\left(j_{1}, j_{2}\right)=\frac{2}{\pi^{2}} \sum_{j=1,3,5, \ldots, \infty} \frac{\nu\left(k_{j}\right)}{j} \times \\
& \times\left(\frac{1}{j-2 j_{1}+2 j_{2}}+\frac{1}{j+2 j_{1}-2 j_{2}}-\right. \\
& \left.-\frac{1}{j-2 j_{1}-2 j_{2}-2}-\frac{1}{j+2 j_{1}+2 j_{2}+2}\right) \text {, }
\end{aligned}
$$


$-c_{0}\left(2 j_{1}+1\right)\left(2 j_{2}+1\right)\left(\frac{a}{L}\right)^{3} \lesssim \frac{S_{29}}{80}<0$.

The values of all $S_{j \geq 2}$ (except for $S_{26}$ and $S_{27}$ ) dependent on one or two parameters $\left(j, l, j_{1}, l_{1} \ldots\right)$ increase in modulus with each parameter, if the parameter is at most $L / a$. If the parameter exceeds $L / a$, then $\left|S_{j}\right|$ almost does not increase. The values of $S_{26}$ and $S_{27}$ increase with the parameter according to (109), (110) for any values of the parameter.

In the above formulae, we use the function

$q(l)=\left[\begin{array}{ll}1 / 2 & l=0, \\ 1 & l=1,2, \ldots, \infty .\end{array}\right.$

The following formulae are also useful for the calculation of sums ( $p, l$, and $j$ are integers):

$$
\begin{aligned}
& \sum_{j= \pm 1, \pm 3, \pm 5, \ldots} \frac{1}{(2 l-j)(2 p-j)}=\frac{\pi^{2}}{4} \delta_{p, l}, \\
& \sum_{j=1,3,5, \ldots}\left(\frac{1}{2 l-j}+\frac{1}{2 l+j}\right) \times \\
\times & \left(\frac{1}{2 p-j}+\frac{1}{2 p+j}\right)=\frac{\pi^{2}}{4}\left(\delta_{p, l}-\delta_{p,-l}\right), \\
p= & \sum_{ \pm 1, \pm 2, \ldots}(2 l+1-2 p)(2 j+1-2 p) \\
& \sum_{p=0,1,2, \ldots}\left(\frac{q(p)}{2 l+1-2 p}+\frac{\pi^{2}}{4} \delta_{j, l},\right. \\
\times & \left(\frac{1}{2 j+1-2 p}+\frac{1}{2 j+1+2 p}\right)= \\
= & \frac{\pi^{2}}{4}\left(\delta_{j, l}-\delta_{2 l+1,-2 j-1}\right) .
\end{aligned}
$$

2) Let us find the quantity $\delta E_{0}(60)$. With the use of the relation $1+\frac{1}{3^{2}}+\frac{1}{5^{2}}+\ldots=\frac{\pi^{2}}{8}$, formula (60) can be written in the form

$$
\begin{aligned}
& \delta E_{0}=\sum_{j=1,3,5, \ldots, \infty}\left|f_{j}\right|^{2} \times \\
& \times \frac{4 K\left(k_{j}\right)\left(n_{0} \nu\left(k_{j}\right)+n_{0} \nu_{0}\right)-\left(n_{0} \nu\left(k_{j}\right)-n_{0} \nu_{0}\right)^{2}}{4 K\left(k_{j}\right)+8 n_{0} \nu\left(k_{j}\right)}= \\
& =\frac{2 n_{0}^{3 / 2} \nu_{0}}{\sqrt{\gamma n}} I(\Upsilon), \\
& I(\Upsilon)=\int_{x_{\min }}^{\infty} \frac{d x}{\pi} \frac{1+\Xi(x)-[\Upsilon x \Xi(x)]^{2} / 8}{x^{2}+\Xi(x)},
\end{aligned}
$$

where $\Upsilon=4 \gamma N_{0} N a^{2} / L^{2}, \Xi(x)=\left(1+\Upsilon x^{2}\right)^{-1}, \gamma=\frac{2 m c_{0}}{\hbar^{2} n}$, $x_{\min }=\frac{1}{2 \sqrt{\Gamma}}$, and potential (98) is used. The weak coupling implies that $\gamma \ll 1$. We find numerically $I\left(\Upsilon<1, x_{\min } \ll 1\right) \approx$ $\approx 1$. For uncharged particles, we have $a \sim L / N, \Upsilon \sim \gamma$. This leads to estimates $I \approx 1$ and $\delta E_{0} \sim 2 n_{0} \nu_{0} / \sqrt{\gamma} \sim E_{0} /(N \sqrt{\gamma})$ for $N^{-2} \ll \gamma \lesssim 1$. That is, $\delta E_{0}$ is negligible for the weak coupling (but for $\gamma \gg N^{-2}$ ), which is consistent with the solutions for point bosons $[7,8,53]$. For $\gamma \lesssim N^{-2}$, the correction $\delta E_{0}$ is not small: $\delta E_{0} \sim N n_{0} \nu_{0} \sim E_{0}$.
1. N.N. Bogoliubov, On the theory of superfluidity. J. Phys. USSR 11, 23 (1947).

2. R. Feynman. Atomic theory of the two-fluid model of liquid helium. Phys. Rev. 94, 262 (1954).

3. N.N. Bogoliubov, D.N. Zubarev. The wave function of the lowest state of a system of interacting Bose particles. Sov. Phys. JETP 1, 83 (1955).

4. K. Brueckner. Theory of Nuclear Structure (Methuen, 1959).

5. E.H. Lieb, W. Liniger. Exact analysis of an interacting Bose gas. I. The general solution and the ground state. Phys. Rev. 130, 1605 (1963).

6. M.D. Tomchenko. Microstructure of He II in the presence of boundaries. Ukr. J. Phys. 59, 123 (2014).

7. M. Gaudin. Boundary energy of a Bose gas in one dimension. Phys. Rev. A 4, 386 (1971).

8. M. Tomchenko. Point bosons in a one-dimensional box: the ground state, excitations and thermodynamics. J. Phys. A: Math. Theor. 48, 365003 (2015).

9. M. Tomchenko. Quasimomentum of an elementary excitation for a system of point bosons with zero boundary conditions. arXiv:1705.10565 [cond-mat.quant-gas].

10. M.A. Cazalilla. Low-energy properties of a one-dimensional system of interacting bosons with boundaries.EPL 59, 793 (2002).

11. M.A. Cazalilla. Bosonizing one-dimensional cold atomic gases. J. Phys. B: At. Mol. Opt. Phys. 37, S1 (2004).

12. M.D. Girardeau, R. Arnowitt. Theory of many-boson systems: Pair theory. Phys. Rev. 113, 755 (1959).

13. N.N. Bogoliubov. Quasi-Averages in Problems of Statistical Mechanics (Dubna report D-781, 1961) (in Russian).

14. N.N. Bogoliubov, Lectures on Quantum Statistics, vol. 2: Quasi-Averages (Gordon and Breach, 1970) [ISBN: 0-67720570-8].

15. A.L. Fetter. Nonuniform states of an imperfect Bose gas. Ann. Phys. 70, 67 (1972).

16. C.W. Gardiner. Particle-number-conserving Bogoliubov method which demonstrates the validity of the time-dependent Gross-Pitaevskii equation for a highly condensed Bose gas. Phys. Rev. A 56, 1414 (1997).

17. M.D. Girardeau. Comment on "Particle-number-conserving Bogoliubov method which demonstrates the validity of the time-dependent Gross-Pitaevskii equation for a highly condensed Bose gas". Phys. Rev. A 58, 775 (1998).

18. A.G. Leggett. Bose-Einstein condensation in the alkali gases: Some fundamental concepts. Rev. Mod. Phys. 73, 307 (2001).

19. V.A. Zagrebnov, J.-B. Bru. The Bogoliubov model of weakly imperfect Bose gas. Phys. Rep. 350, 291 (2001).

20. V.A. Zagrebnov. The Bogoliubov theory of weakly imperfect Bose gas and its modern development in: N.N. Bogoliubov, Collection of scientific works in 12 volumes, ed. by A.D. Sukhanov (Nauka, 2007), v. 8. (in Russian)[ISBN: 978-5020339422, 978-5-02-035723-5].

21. A. Rovenchak. Weakly-interacting bosons in a trap within approximate second quantization approach. J. Low Temp. Phys. 148, 411 (2007).

ISSN 2071-0194. Ukr. J. Phys. 2019. Vol. 64, No. 3 
22. A. Rovenchak. Effective Hamiltonian and excitation spectrum of harmonically trapped bosons. Low Temp. Phys. 42, 36 (2016)

23. V.B. Bobrov, A.G. Zagorodny, S.A. Trigger. Coulomb interaction potential and Bose-Einstein condensate. Low Temp. Phys. 41, 901 (2015).

24. J. Sato, E. Kaminishi, T. Deguchi. Finite-size scaling behavior of Bose-Einstein condensation in the 1D Bose gas. arXiv:1303.2775 [cond-mat.quant-gas].

25. J. Grond, A.I. Streltsov, A.U.J. Lode, K. Sakmann, L.S. Cederbaum, O.E. Alon. Excitation spectra of manybody systems by linear response: General theory and applications to trapped condensates. Phys. Rev. A 88, 023606 (2013).

26. J.W. Kane, L.P. Kadanoff. Long-range order in superfluid helium. Phys. Rev. 155, 80 (1967).

27. P.C. Hohenberg. Existence of long-range order in one and two dimensions. Phys. Rev. 158, 383 (1967).

28. U.R. Fischer. Existence of long-range order for trapping interacting bosons. Phys. Rev. Lett. 89, 280402 (2002).

29. A.I. Bugrij, V.M. Loktev. On the theory of Bose-Einstein condensation of quasiparticles: On the possibility of condensation of ferromagnons at high temperatures. Low Temp. Phys. 33, 37 (2007).

30. D.A. Kirzhnits. Superconductivity and elementary particles. Sov. Phys. Usp. 21, 470 (1978).

31. A. Griffin. BEC and the new world of coherent matter waves, in Theoretical Physics at the End of the Twentieth Century, ed. by Y. Saint-Aubin and L. Vinet (Springer, 2002). [ISBN: 0387953116, 978-0387953113].

32. O. Penrose, L. Onsager. Bose-Einstein condensation and liquid helium. Phys. Rev. 104, 576 (1956).

33. M. Tomchenko. On a fragmented condensate in a uniform Bose system. arXiv:1808.08203 [cond-mat.quant-gas].

34. N.N. Bogoliubov. Lectures on Quantum Statistics, vol. 1: Quantum Statistics (Gordon and Breach, 1967) [ISBN: 0677200307, 9780677200309].

35. E.P. Gross. Unified theory of interacting bosons. Phys. Rev. 106, 161 (1957).

36. E.P. Gross. Structure of a quantized vortex in boson systems. Nuovo Cimento 20, 454 (1961).

37. L.P. Pitaevskii. Vortex lines in an imperfect Bose gas. Sov Phys. JETP 13, 451 (1961).

38. M. Tomchenko. Expansions of the interatomic potential under various boundary conditions and the transition to the thermodynamic limit. arXiv:1403.8014 [condmat.other].

39. S.N. Bose. Plancks gesetz und lichtquantenhypothese. $Z$. Phys. 26, 178 (1924).

40. C.J. Pethick, H. Smith. Bose-Einstein Condensation in Dilute Gases (Cambridge Univ. Press, 2008), Chap. 15.

41. W. Ketterle, N.J. van Druten. Bose-Einstein condensation of a finite number of particles trapped in one or three dimensions. Phys. Rev. A 54, 656 (1996).

42. M. Tomchenko. Bose-Einstein condensation in a onedimensional system of interacting bosons. J. Low Temp. Phys. 182, 170 (2016).

43. M. Schwartz. Off-diagonal long-range behavior of interacting Bose systems. Phys. Rev. B 15, 1399 (1977).
44. F.D.M. Haldane. Effective harmonic-fluid approach to lowenergy properties of one-dimensional quantum fluids. Phys. Rev. Lett. 47, 1840 (1981).

45. D.S. Petrov, D.M. Gangardt, G.V. Shlyapnikov. Low-dimensional trapped gases. J. Phys. IV France 116, 3 (2004).

46. V.N. Popov. On the theory of the superfluidity of two- and one-dimensional bose systems. Theor. Math. Phys. 11, 565 (1972).

47. A. Berkovich, G. Murthy. Time-dependent multipoint correlation functions of the nonlinear Schrödinger model. Phys. Lett. A 142, 121 (1989).

48. C. Mora, Y. Castin. Extension of Bogoliubov theory to quasicondensates. Phys. Rev. A 67, 053615 (2003).

49. V. Dunjko, M. Olshanii. A Hermite-Padé perspective on Gell-Mann-Low renormalization group: an application to the correlation function of Lieb-Liniger gas. arXiv:0910.0565 [cond-mat.quant-gas].

50. I. Bouchoule, N.J. van Druten, C.I. Westbrook. Atom chips and one-dimensional Bose gases. arXiv:0901.3303 [physics.atom-ph].

51. G.E. Astrakharchik, S. Giorgini. Correlation functions of a Lieb-Liniger Bose gas. J. Phys. B: At. Mol. Opt. Phys. 39, S1 (2006).

52. J.-S. Caux, P. Calabrese, N.A. Slavnov. One-particle dynamical correlations in the one-dimensional Bose gas. J. Stat. Mech. P01008 (2007).

53. M.T. Batchelor, X.W. Guan, N. Oelkers, C. Lee. The 1D interacting Bose gas in a hard wall box. J. Phys. A: Math. Gen. 38, 7787 (2005).

54. T. Giamarchi. Quantum Physics in One Dimension (Clarendon Press, 2003) [ISBN: 0-19-852500-1].

55. J.M. Vogels, K. Xu, C. Raman, J.R. Abo-Shaeer, W. Ketterle. Experimental observation of the Bogoliubov transformation for a Bose-Einstein condensed gas. Phys. Rev. Lett. 88, 060402 (2002).

Received 04.11.18

М.Д. Томченко

\section{НИЖНІ ЕНЕРГЕТИЧНІ РІВНI}

\section{ОДНОВИМІРНОГО СЛАБКО ВЗАЕМОДІЮЧОГО} БОЗЕ-ГАЗУ З НУЛЬОВИМИ МЕЖКОВИМИ УМОВАМИ

$\mathrm{P}$ е $з$ ю м е

Ми діагоналізували вторинно квантований гамільтоніан одновимірного бозе-газу для відштовхувального міжатомного потенціалу загального вигляду та нульових межових умов. При малій константі зв'язку розв'язки для енергії основного стану $E_{0}$ та закону дисперсії $E(k)$ збігаються з відомими розв'язками для періодичної системи. При цьому одночастинкова матриця густини $F_{1}\left(x, x^{\prime}\right)$ є близькою до розв'язку для періодичної системи, якщо $T=0$, та помітно відрізняється від останнього при $T>0$. Також ми отримали, що хвильова функція $\langle\hat{\psi}(x, t)\rangle$ ефективного конденсату близька до константи $\sqrt{N_{0} / L}$ всередині системи та обертається на нуль на межах (тут $N_{0}$ - число атомів у конденсаті, $L$ - розмір системи). Ми знайшли критерій застосовності методу, згідно з яким метод працює для скінченної системи з малою константою зв'язку (слабка взаємодія або велика концентрація) та дуже малою температурою. 